\title{
Interpolation and extrapolation of smooth functions by linear operators
}

Charles Fefferman

\begin{abstract}
Let $C^{m, 1}\left(\mathbb{R}^{n}\right)$ be the space of functions on $\mathbb{R}^{n}$ whose $m^{\text {th }}$ derivatives are Lipschitz 1. For $E \subset \mathbb{R}^{n}$, let $C^{m, 1}(E)$ be the space of all restrictions to $E$ of functions in $C^{m, 1}\left(\mathbb{R}^{n}\right)$. We show that there exists a bounded linear operator $T: C^{m, 1}(E) \rightarrow C^{m, 1}\left(\mathbb{R}^{n}\right)$ such that, for any $f \in C^{m, 1}(E)$, we have $T f=f$ on $E$.
\end{abstract}

\section{Introduction}

Fix $m, n \geq 1$. In [8], we studied the following problems:

Large Finite Problem: Given a finite set $E \subset \mathbb{R}^{n}$ and functions $f: E \rightarrow \mathbb{R}$ and $\sigma: E \rightarrow[0, \infty)$, find the least $M>0$ for which there exists $F \in C^{m}\left(\mathbb{R}^{n}\right)$, satisfying $\|F\|_{C^{m}\left(\mathbb{R}^{n}\right)} \leq M$, and $|F(x)-f(x)| \leq M \cdot \sigma(x)$ for all $x \in E$.

Infinite Problem: Given an arbitrary set $E \subset \mathbb{R}^{n}$ and functions $f: E \rightarrow \mathbb{R}$ and $\sigma: E \rightarrow[0, \infty)$, decide whether there exist a function $F \in C^{m-1,1}\left(\mathbb{R}^{n}\right)$ and a finite constant $M$, satisfying

(0.1) $\|F\|_{C^{m-1,1}\left(\mathbb{R}^{n}\right)} \leq M \quad$ and $\quad|F(x)-f(x)| \leq M \cdot \sigma(x) \quad$ for all $x \in E$.

In the special case $\sigma \equiv 0$, the Infinite Problem amounts to deciding whether a given function $f: E \rightarrow \mathbb{R}$ extends to a $C^{m-1,1}$ function on all of $\mathbb{R}^{n}$. (As usual, $C^{m-1,1}\left(\mathbb{R}^{n}\right)$ denotes the space of functions whose $(m-1)^{\text {rst }}$ derivatives are Lipschitz 1.) This is a variant of a classical problem of Whitney [18]. Important work on closely related questions was done by Whitney $[17,18,19]$, Glaeser [9], Brudnyi and Shvartsman [2-6, 12, 13, 14], and Bierstone-MilmanPawlucki [1], as explained partially in [8].

2000 Mathematics Subject Classification: 49K24, 52A35.

Keywords: Whitney extension problem, linear operators. 
In this paper, we will show that an essentially optimal $M$ may be achieved for the two problems above, by taking $F$ to depend linearly on $f$. More precisely, for the Large Finite Problem, we have the following result.

Theorem 1 Let $E \subset \mathbb{R}^{n}$ be finite, and let $\sigma: E \longrightarrow[0, \infty)$ be given. Let $C(E)$ be the vector space of (real-valued) functions on $E$. Then there exists a linear map $T: C(E) \longrightarrow C^{m}\left(\mathbb{R}^{n}\right)$, with the following property:

Let $f \in C(E)$ be given. Assume there exists $F \in C^{m}\left(\mathbb{R}^{n}\right)$, with

$$
\|F\|_{C^{m}\left(\mathbb{R}^{n}\right)} \leq 1 \quad \text { and with } \quad|F(x)-f(x)| \leq \sigma(x) \quad \text { for all } x \in E .
$$

Then we have

$$
\|T f\|_{C^{m}\left(\mathbb{R}^{n}\right)} \leq A \quad \text { and } \quad|T f(x)-f(x)| \leq A \cdot \sigma(x) \quad \text { for all } x \in E,
$$

for a constant $A$ depending only on $m$ and $n$.

For the Infinite Problem, we introduce a Banach space $C^{m-1,1}(E, \sigma)$ associated to an arbitrary set $E \subset \mathbb{R}^{n}$ and a function $\sigma: E \longrightarrow[0, \infty)$. This space consists of all functions $f: E \longrightarrow \mathbb{R}$ for which there exist $F \in C^{m-1,1}\left(\mathbb{R}^{n}\right)$ and $M<\infty$ satisfying (0.1). The norm $\|f\|_{C^{m-1,1}(E, \sigma)}$ is defined as the infimum of all possible $M$ in (0.1).

Our result for the Infinite Problem is as follows:

Theorem 2 Let $E \subset \mathbb{R}^{n}$ be an arbitrary subset, and let $\sigma: E \longrightarrow[0, \infty)$ be given. Then there exists a linear map $T: C^{m-1,1}(E, \sigma) \longrightarrow C^{m-1,1}\left(\mathbb{R}^{n}\right)$, with the following property:

$$
\begin{aligned}
& \text { Let } f \in C^{m-1,1}(E, \sigma) \text { be given with }\|f\|_{C^{m-1,1}(E, \sigma)} \leq 1 \text {. Then we have } \\
& \|T f\|_{C^{m-1,1}\left(\mathbb{R}^{n}\right)} \leq A \quad \text { and }|T f(x)-f(x)| \leq A \cdot \sigma(x) \quad \text { for all } x \in E
\end{aligned}
$$

for a constant $A$ depending only on $m$ and $n$.

One of the conjectures of Brudnyi and Shvartsman in [4] is closely analogous to our Theorems 1 and 2. One of their theorems [5] includes the case $\sigma=0, m=2$ of our results as a special case. I am grateful to Brudnyi and Shvartsman for raising with me the issue of linear dependence of $F$ on $f$ above, and also to E. Bierstone and P. Milman for valuable discussions.

An interesting refinement of Theorem 1 concerns operators of "bounded depth". We say that an operator $T: C(E) \longrightarrow C^{m}\left(\mathbb{R}^{n}\right)$ has "bounded depth" if every point of $\mathbb{R}^{n}$ has a neighborhood $U$, for which $\left.T f\right|_{U}$ depends only on $\left.f\right|_{S}$ for a subset $S \subset E$, with \# $\#$ (S) bounded a-priori in terms of $m$ and $n$. (Here, $\#(S)$ denotes the number of points in $S$.) The operator $T$ in the conclusion of Theorem 1 may be taken to have bounded depth. This follows without difficulty from our proof of Theorem 1, but we omit the details. 
Most of our proof of Theorem 1 repeats ideas in [8] with straightforward modifications. However, we need one additional idea, which we now sketch.

In [8], we introduced the sets

$$
\begin{array}{r}
\mathcal{K}_{f}(y ; S, C)=\left\{J_{y}(F): F \in C^{m}\left(\mathbb{R}^{n}\right),\|F\|_{C^{m}\left(\mathbb{R}^{n}\right)} \leq C,\right. \\
|F(x)-f(x)| \leq C \cdot \sigma(x) \text { on } S\}
\end{array}
$$

for $y \in \mathbb{R}^{n}, S \subset E$. Here, and throughout this paper, $J_{y}(F)$ denotes the $(m-1)$-jet of $F$ at $y$.

A crucial point in [8] was to show that, for suitable $k^{\#}$ depending only on $m$ and $n$, there is an $(m-1)^{\text {rst }}$ degree polynomial $P$ belonging to $\mathcal{K}_{f}(y ; S, C)$ for all $S \subset E$ having at most $k^{\#}$ elements. The set of all such $P$ was called $\mathcal{K}_{f}\left(y ; k^{\#}, C\right)$ in [8].

Roughly speaking, any polynomial in $\mathcal{K}_{f}\left(y ; k^{\#}, C\right)$ is a plausible guess for the $(m-1)$-jet at $y$ of the function $T f$ in Theorem 1 .

To prove Theorem 1 , we must not only show that $\mathcal{K}_{f}\left(y ; k^{\#}, C\right)$ is nonempty; we must produce a $P \in \mathcal{K}_{f}\left(y ; k^{\#}, C\right)$ that depends linearly on $f$. Once this is done, we can essentially repeat the arguments in [8] for large finite sets $E$ and strictly positive $\sigma$, because all the functions $F \in C^{m}\left(\mathbb{R}^{n}\right)$ constructed in [8] depend linearly on $f$ and $P$.

To find $P \in \mathcal{K}_{f}\left(y ; k^{\#}, C\right)$ depending linearly on $f$, we introduce the auxiliary convex sets

$$
\Gamma(y, S)=\left\{J_{y}(\varphi):\|\varphi\|_{C^{m}\left(\mathbb{R}^{n}\right)} \leq 1 \text { and }|\varphi(x)| \leq \sigma(x) \text { on } S\right\} .
$$

By using elementary properties of convex sets, reminiscent of our applications of Helly's theorem in [8], we show that there exists a subset $S^{y} \subset E$, with the following properties:

(a) The number of points in $S^{y}$ is bounded by a constant depending only on $m$ and $n$; and

(b) Any polynomial $P \in \Gamma\left(y, S^{y}\right)$ belongs also to $C \cdot \Gamma(y, S)$, for any $S \subset E$ with at most $k^{\#}$ points, where $C$ is a constant depending only on $m$ and $n$. The set $S^{y}$ depends only on the set $E$ and the function $\sigma$, not on $f$.

Because $S^{y}$ contains only a few points (property (a) above), it is easy to fit a function $F \in C^{m}\left(\mathbb{R}^{n}\right)$ to $f$ on $S^{y}$, with $F$ depending linearly on $f$. We may then simply define $P$ to be the $(m-1)$-jet of $F$ at $y$. Thus, $P$ depends linearly on $f$. Thanks to property (b) above, we can show also that $P$ belongs to $\mathcal{K}_{f}\left(y ; k^{\#}, C\right)$. This argument appears in Section 10 below, in a lightly disguised form that doesn't explicitly mention $F$. (The minimization of the quadratic form in Section 10 is morally equivalent to finding $F$ as sketched above, as we see from the standard Whitney extension theorem.) 
Once Theorem 1 is established, it isn't hard to deduce Theorem 2. We proceed by applying Theorem 1 to arbitrarily large finite subsets of $E$, and then passing to a Banach limit. (We recall Banach limits in Section 15 below.)

We now begin the proofs of Theorems 1 and 2. Unfortunately, we assume from here on that the reader is thoroughly familiar with [8].

\section{Notation}

Fix $m, n \geq 1$ throughout this paper.

$C^{m}\left(\mathbb{R}^{n}\right)$ denotes the space of functions $F: \mathbb{R}^{n} \rightarrow \mathbb{R}$ whose derivatives of order $\leq m$ are continuous and bounded on $\mathbb{R}^{n}$. For $F \in C^{m}\left(\mathbb{R}^{n}\right)$, we define $\|F\|_{C^{m}\left(\mathbb{R}^{n}\right)}=\sup _{x \in \mathbb{R}^{n}} \max _{|\beta| \leq m}\left|\partial^{\beta} F(x)\right| \quad$ and $\quad\left\|\partial^{m} F\right\|_{C^{0}\left(\mathbb{R}^{n}\right)}=\sup _{x \in \mathbb{R}^{n}} \max _{|\beta|=m}\left|\partial^{\beta} F(x)\right|$.

For $F \in C^{m}\left(\mathbb{R}^{n}\right)$ and $y \in \mathbb{R}^{n}$, we define $J_{y}(F)$ to be the $(m-1)$-jet of $F$ at $y$, i.e., the polynomial

$$
J_{y}(F)(x)=\sum_{|\beta| \leq m-1} \frac{1}{\beta !}\left(\partial^{\beta} F(y)\right) \cdot(x-y)^{\beta} .
$$

$C^{m-1,1}\left(\mathbb{R}^{n}\right)$ denotes the space of all functions $F: \mathbb{R}^{n} \rightarrow \mathbb{R}$, whose derivatives of order $\leq m-1$ are continuous, and for which the norm

$$
\|F\|_{C^{m-1,1}\left(\mathbb{R}^{n}\right)}=\max _{|\beta| \leq m-1}\left\{\sup _{x \in \mathbb{R}^{n}}\left|\partial^{\beta} F(x)\right|+\sup _{\substack{x, y \in \mathbb{R}^{n} \\ x \neq y}} \frac{\left|\partial^{\beta} F(x)-\partial^{\beta} F(y)\right|}{|x-y|}\right\}
$$

is finite.

Let $\mathcal{P}$ denote the vector space of polynomials of degree $\leq m-1$ on $\mathbb{R}^{n}$ (with real coefficients), and let $D$ denote the dimension of $\mathcal{P}$.

Let $\mathcal{M}$ denote the set of all multi-indices $\beta=\left(\beta_{1}, \ldots, \beta_{n}\right)$ with $|\beta|=$ $\beta_{1}+\cdots+\beta_{n} \leq m-1$. Let $\mathcal{M}^{+}$denote the set of multi-indices $\beta=\left(\beta_{1}, \ldots, \beta_{n}\right)$ with $|\beta| \leq m$.

If $\alpha$ and $\beta$ are multi-indices, then $\delta_{\beta \alpha}$ denotes the Kronecker delta, equal to 1 if $\beta=\alpha$ and 0 otherwise.

We will be dealing with functions of $x$ parametrized by $y\left(x, y \in \mathbb{R}^{n}\right)$. We will often denote these by $\varphi^{y}(x)$, or by $P^{y}(x)$ in case $x \mapsto P^{y}(x)$ is a polynomial for fixed $y$. When we write $\partial^{\beta} P^{y}(y)$, we always mean the value of $\left(\frac{\partial}{\partial x}\right)^{\beta} P^{y}(x)$ at $x=y$; we never use $\partial^{\beta} P^{y}(y)$ to denote the derivative of order $\beta$ of the function $y \mapsto P^{y}(y)$.

We write $B(x, r)$ to denote the ball with center $x$ and radius $r$ in $\mathbb{R}^{n}$. If $Q$ is a cube in $\mathbb{R}^{n}$, then $\delta_{Q}$ denotes the diameter of $Q$; and $Q^{\star}$ denotes the cube whose center is that of $Q$, and whose diameter is 3 times that of $Q$. 
If $Q$ is a cube in $\mathbb{R}^{n}$, then to "bisect" $Q$ is to partition it into $2^{n}$ congruent subcubes in the obvious way. Later on, we will fix a cube $Q^{\circ} \subset \mathbb{R}^{n}$, and define the class of "dyadic" cubes to consist of $Q^{\circ}$, together with all the cubes arising from $Q^{\circ}$ by repeated bisection. Each dyadic cube $Q$ other than $Q^{\circ}$ arises from bisecting a dyadic cube $Q^{+} \subseteq Q^{\circ}$, with $\delta_{Q^{+}}=2 \delta_{Q}$. We call $Q^{+}$the dyadic "parent" of $Q$. Note that $Q^{+} \subset Q^{\star}$.

For any finite set $X$, write $\#(X)$ to denote the number of elements of $X$. If $X$ is infinite, then we define $\#(X)=\infty$.

Let $E \subset \mathbb{R}^{n}$ and $\sigma: E \longrightarrow[0, \infty)$ be given. Then, as in the Introduction, $C^{m-1,1}(E, \sigma)$ denotes the space of all functions $f: E \longrightarrow \mathbb{R}$, for which there exist $M>0, F \in C^{m-1,1}\left(\mathbb{R}^{n}\right)$, with

(a) $\|F\|_{C^{m-1,1}\left(\mathbb{R}^{n}\right)} \leq M$ and

(b) $|F(x)-f(x)| \leq M \sigma(x)$ for all $x \in E$.

The norm $\|f\|_{C^{m-1,1}(E, \sigma)}$ is defined as the infimum of the set of all $M>0$ for which there exists an $F$ satisfying (a) and (b).

Similarly, $C^{m}(E, \sigma)$ denotes the space of all functions $f: E \longrightarrow \mathbb{R}$, for which there exist $M>0, F \in C^{m}\left(\mathbb{R}^{n}\right)$, with

(c) $\|F\|_{C^{m}\left(\mathbb{R}^{n}\right)} \leq M$ and

(d) $|F(x)-f(x)| \leq M \sigma(x)$ for all $x \in E$.

The norm $\|f\|_{C^{m}(E, \sigma)}$ is defined as infimum of all $M>0$ for which there exists $F$ satisfying (c) and (d).

Suppose $E \subset \mathbb{R}^{n}$ (finite), $\sigma: E \rightarrow(0, \infty)$ and $\delta>0$ are given. If $f: E \rightarrow \mathbb{R}$, then the norm $\|f\|_{C^{m}(E, \sigma ; \delta)}$ is defined as the infimum of all $M>0$ for which there exists $F \in C^{m}\left(\mathbb{R}^{n}\right)$, with $\left\|\partial^{\beta} F\right\|_{C^{0}\left(\mathbb{R}^{n}\right)} \leq M \delta^{-|\beta|}$ for $|\beta| \leq m$, and

$$
|F(x)-f(x)| \leq M \delta^{-m} \sigma(x) \text { for all } x \in E .
$$

We write $C^{m}(E, \sigma ; \delta)$ for the space of all functions $f: E \rightarrow \mathbb{R}$, equipped with the above norm.

If $\delta>0$ and $F \in C^{m}\left(\mathbb{R}^{n}\right)$, then we define

$$
\|F\|_{C^{m}\left(\mathbb{R}^{n} ; \delta\right)}=\max _{|\beta| \leq m} \delta^{|\beta|}\left\|\partial^{\beta} F\right\|_{C^{0}\left(\mathbb{R}^{n}\right)} .
$$

We write $C^{m}\left(\mathbb{R}^{n} ; \delta\right)$ for the space $C^{m}\left(\mathbb{R}^{n}\right)$ equipped with the norm $\|F\|_{C^{m}\left(\mathbb{R}^{n} ; \delta\right)}$.

If $E \subset \mathbb{R}^{n}$ is finite, then $C^{0}(E)$ denotes the space of functions $f: E \rightarrow \mathbb{R}$, equipped with the norm $\|f\|_{C^{0}(E)}=\max _{x \in E}|f(x)|$.

A subset $\mathcal{K} \subset \mathbb{R}^{d}$ is called symmetric if, for any $x \in \mathbb{R}^{d}, x \in \mathcal{K}$ implies $-x \in \mathcal{K}$. If $\mathcal{K} \subset \mathbb{R}^{d}$ is symmetric, and if $C>0$ is given then $C \mathcal{K}$ denotes the set of all the points $C x \in \mathbb{R}^{d}$ with $x \in \mathcal{K}$. 


\section{Sharp Whitney}

One of the main results of [8] is as follows.

Sharp Whitney Theorem for Finite Sets. Given $m, n \geq 1$, there exist constants $k_{s w}^{\#}(m, n)$ and $A(m, n)$, depending only on $m$ and $n$, for which the following holds.

Let $E \subset \mathbb{R}^{n}$ be finite, and let $f: E \rightarrow \mathbb{R}, \sigma: E \rightarrow[0, \infty)$ be functions on $E$. Assume that, given any $S \subset E$ with $\#(S) \leq k_{s w}^{\#}(m, n)$, there exists $F^{S} \in C^{m}\left(\mathbb{R}^{n}\right)$, with $\left\|F^{S}\right\|_{C^{m}\left(\mathbb{R}^{n}\right)} \leq 1$ and $\left|F^{S}(x)-f(x)\right| \leq \sigma(x)$ for all $x \in S$.

Then there exists $F \in C^{m}\left(\mathbb{R}^{n}\right)$, with

$\|F\|_{C^{m}\left(\mathbb{R}^{n}\right)} \leq A(m, n) \quad$ and $\quad|F(x)-f(x)| \leq A(m, n) \cdot \sigma(x)$ for all $x \in E$.

In terms of the spaces $C^{m}(E, \sigma ; \delta)$, we have the following

Corollary. Given $m, n \geq 1$, there exist constants $k_{s w}^{\#}(m, n)$ and $A(m, n)$, depending only on $m$ and $n$, for which the following holds.

Let $E \subset \mathbb{R}^{n}$ be finite, let $f: E \longrightarrow \mathbb{R}$, let $\sigma: E \longrightarrow[0, \infty)$, and let $\delta>0$. Then

$$
\|f\|_{C^{m}(E, \sigma ; \delta)} \leq A(m, n) \cdot \max \left\{\|f\|_{C^{m}\left(S,\left.\sigma\right|_{S} ; \delta\right)}: S \subset E, \#(S) \leq k_{s w}^{\#}(m, n)\right\} .
$$

Proof. The case $\delta=1$ is immediate from Sharp Whitney for Finite Sets; the general case follows by rescaling.

\section{A Lemma on Convex Sets}

The following result is surely known (probably in sharper form) but I haven't found it in the literature.

Lemma on Convex Sets. Let $\mathcal{F}$ be a finite collection of compact, convex, symmetric subsets of $\mathbb{R}^{D}$. Suppose 0 is an interior point of each $\mathcal{K} \in \mathcal{F}$. Then, with $C_{D}$ depending only on $D$, and with $\ell=D \cdot(D+1)$, there exist $\mathcal{K}_{1}, \ldots, \mathcal{K}_{\ell} \in \mathcal{F}$, with

$$
\mathcal{K}_{1} \cap \cdots \cap \mathcal{K}_{\ell} \subset C_{D} \cdot\left(\bigcap_{\mathcal{K} \in \mathcal{F}} \mathcal{K}\right) .
$$

Proof. We use the following standard results on convex sets.

Helly's Theorem ([16]). Let $\mathcal{F}$ be any collection of compact, convex subsets of $\mathbb{R}^{D}$. If the intersection of all the sets $\mathcal{K} \in \mathcal{F}$ is empty, then already the intersection of some $(D+1)$ sets $\mathcal{K}_{1}, \ldots, \mathcal{K}_{D+1} \in \mathcal{F}$ is empty. 
Fritz John's Lemma (See the simple proof by A. Córdoba and A. Gallego in [10]). Let $\mathcal{K} \subset \mathbb{R}^{D}$ be any bounded, symmetric, convex set with non-empty interior. Then there exists an ellipsoid $E \subset \mathbb{R}^{D}$, centered at the origin, with $E \subset \mathcal{K} \subset C_{D} E$, where $C_{D}$ depends only on $D$.

Proof of the Lemma on Convex Sets. Let $\mathcal{K}^{*}$ be the intersection of all the sets $\mathcal{K} \in \mathcal{F}$. Then $\mathcal{K}^{*}$ is compact, convex, symmetric, and contains 0 as an interior point. Applying the Lemma of F. John, we obtain an ellipsoid $E \subset \mathbb{R}^{D}$, centered at the origin, with $E \subset \mathcal{K}^{*} \subset C_{D} \cdot E$. Applying a linear transformation to $\mathbb{R}^{D}$, we may assume without loss of generality that $E$ is the unit ball. Hence, for constants $c_{D}, C_{D}^{\prime}$, depending only on $D$, we have

$$
c_{D} Q \subset \mathcal{K}^{*} \subset C_{D}^{\prime} Q
$$

$$
\text { with } Q=\left\{x=\left(x_{1}, \ldots, x_{D}\right) \in \mathbb{R}^{D}:\left|x_{j}\right|<1 \text { for each } j\right\} \text {. }
$$

Given $\mathcal{K} \in \mathcal{F}$ and $1 \leq j \leq D$, we set

$$
\operatorname{Cap}(\mathcal{K}, j)=\left\{\left(x_{1}, \ldots, x_{D}\right) \in \mathcal{K}: x_{j} \geq C_{D}^{\prime}\right\} .
$$

Each $\operatorname{Cap}(\mathcal{K}, j)$ is a compact, convex subset of $\mathbb{R}^{D}$. Moreover, since $\mathcal{K}^{*} \subset$ $C_{D}^{\prime} Q$, we know that the intersection of all the $\operatorname{sets} \operatorname{Cap}(\mathcal{K}, j)(\mathcal{K} \in \mathcal{F})$ is empty, for each fixed $j$. Applying Helly's Theorem to the $\operatorname{Cap}(\mathcal{K}, j)$, we obtain sets $\mathcal{K}_{1}^{(j)}, \ldots, \mathcal{K}_{D+1}^{(j)} \in \mathcal{F}$, for which the intersection of $\operatorname{Cap}\left(\mathcal{K}_{i}^{(j)}, j\right)$ over $i=1, \ldots, D+1$ is empty. This means that every $x=\left(x_{1}, \ldots, x_{D}\right) \in$ $\mathcal{K}_{1}^{(j)} \cap \cdots \cap \mathcal{K}_{D+1}^{(j)}$ satisfies $x_{j}<C_{D}^{\prime}$. Since the $\mathcal{K}_{i}^{(j)}$ are symmetric, we have $\left|x_{j}\right|<C_{D}^{\prime}$ for all

$$
x=\left(x_{1}, \ldots, x_{D}\right) \in \bigcap_{i=1}^{D+1} \mathcal{K}_{i}^{(j)} .
$$

Consequently, the intersection

$$
\bigcap_{j=1}^{D} \bigcap_{i=1}^{D+1} \mathcal{K}_{i}^{(j)}
$$

is contained in $C_{D}^{\prime} Q$, which in turn is contained in

$$
\left(C_{D}^{\prime} / C_{D}\right) \cdot \bigcap_{\mathcal{K} \in \mathcal{F}} \mathcal{K}
$$

thanks to (3.1). Thus, we have found $D \cdot(D+1)$ sets $\mathcal{K}_{i}^{(j)} \in \mathcal{F}$, whose intersection is contained in

$$
C_{D}^{\prime \prime} \cdot \bigcap_{\mathcal{K} \in \mathcal{F}} \mathcal{K}
$$

The proof of the Lemma is complete. 


\section{Statement of the Main Lemmas}

Fix $\mathcal{A} \subset \mathcal{M}$. We state two results involving $\mathcal{A}$. For the second result, we use an order relation between multi-indices, defined in [8] and denoted by $>$.

Weak Main Lemma for $\mathcal{A}$. Given $m, n \geq 1$, there exist constants $k^{\#}, a_{0}$, depending only on $m$ and $n$, for which the following holds.

Suppose we are given a finite set $E \subset \mathbb{R}^{n}$ and a function $\sigma: E \rightarrow(0, \infty)$. Suppose we are also given a point $y^{0} \in \mathbb{R}^{n}$ and a family of polynomials $P_{\alpha} \in \mathcal{P}$, indexed by $\alpha \in \mathcal{A}$. Assume that the following conditions are satisfied:

(WL1) $\partial^{\beta} P_{\alpha}\left(y^{0}\right)=\delta_{\beta \alpha}$ for all $\beta, \alpha \in \mathcal{A}$.

(WL2) $\left|\partial^{\beta} P_{\alpha}\left(y^{0}\right)-\delta_{\beta \alpha}\right| \leq a_{0}$ for all $\alpha \in \mathcal{A}, \beta \in \mathcal{M}$.

(WL3) Given $S \subset E$ with $\#(S) \leq k^{\#}$, and given $\alpha \in \mathcal{A}$, there exists $\varphi_{\alpha}^{S} \in C^{m}\left(\mathbb{R}^{n}\right)$, with

(a) $\left\|\partial^{m} \varphi_{\alpha}^{S}\right\|_{C^{0}\left(\mathbb{R}^{n}\right)} \leq a_{0}$.

(b) $\left|\varphi_{\alpha}^{S}(x)\right| \leq C \sigma(x)$ for all $x \in S$.

(c) $J_{y^{0}}\left(\varphi_{\alpha}^{S}\right)=P_{\alpha}$.

Then there exists a linear operator $\mathcal{E}: C^{m}(E, \sigma) \longrightarrow C^{m}\left(\mathbb{R}^{n}\right)$, satisfying the following conditions:

(WL4) $\mathcal{E}$ has norm at most $C^{\prime}$;

(WL5) $|\mathcal{E} f(x)-f(x)| \leq C^{\prime} \cdot\|f\|_{C^{m}(E, \sigma)} \cdot \sigma(x)$ for all $f \in C^{m}(E, \sigma)$ and $x \in E \cap B\left(y^{0}, c^{\prime}\right)$. Here, $c^{\prime}$ and $C^{\prime}$ in $(\mathbf{W L 4}, 5)$ depend only on $C, m, n$ in $(\mathrm{WL} 1,2,3)$.

Strong Main Lemma for $\mathcal{A}$. Given $m, n \geq 1$, there exists $k^{\#}$, depending only on $m$ and $n$, for which the following holds.

Suppose we are given a finite set $E \subset \mathbb{R}^{n}$, and a function $\sigma: E \rightarrow(0, \infty)$. Suppose we are also given a point $y^{0} \in \mathbb{R}^{n}$, and a family of polynomials $P_{\alpha} \in \mathcal{P}$, indexed by $\alpha \in \mathcal{A}$. Assume that the following conditions are satisfied:

(SL1) $\partial^{\beta} P_{\alpha}\left(y^{0}\right)=\delta_{\beta \alpha}$ for all $\alpha, \beta \in \mathcal{A}$.

(SL2) $\left|\partial^{\beta} P_{\alpha}\left(y^{0}\right)\right| \leq C$ for all $\alpha \in \mathcal{A}, \beta \in \mathcal{M}$ with $\beta \geq \alpha$. 
(SL3) Given $S \subset E$ with $\#(S) \leq k^{\#}$ and given $\alpha \in \mathcal{A}$, there exists $\varphi_{\alpha}^{S} \in$ $C^{m}\left(\mathbb{R}^{n}\right)$ with

(a) $\left\|\partial^{m} \varphi_{\alpha}^{S}\right\|_{C^{0}\left(\mathbb{R}^{n}\right)} \leq C$,

(b) $\left|\varphi_{\alpha}^{S}(x)\right| \leq C \sigma(x)$ for all $x \in S$,

(c) $J_{y^{0}}\left(\varphi_{\alpha}^{S}\right)=P_{\alpha}$.

Then there exists a linear operator $\mathcal{E}: C^{m}(E, \sigma) \longrightarrow C^{m}\left(\mathbb{R}^{n}\right)$, satisfying the following conditions:

(SL4) $\mathcal{E}$ has norm at most $C^{\prime}$;

(SL5) $|\mathcal{E} f(x)-f(x)| \leq C^{\prime}\|f\|_{C^{m}(E, \sigma)} \cdot \sigma(x)$ for all $f \in C^{m}(E, \sigma)$ and all $x \in E \cap B\left(y^{0}, c^{\prime}\right)$. Here, $c^{\prime}$ and $C^{\prime}$ in $(\mathbf{S L 4}, \mathbf{5})$ depend only on $C, m, n$ in $(\mathrm{SL} 1,2,3)$.

\section{Plan of the Proof}

Recall from [8] that subsets of $\mathcal{M}$ are totally ordered by a relation denoted by $<$. As in [8], we will establish the WEAK and Strong MAIN LEMmas for any $\mathcal{A} \subset \mathcal{M}$, by proving the following results.

Lemma PP1. The WEAK and Strong MAIn LEMmas both hold for $\mathcal{A}=\mathcal{M}$. Lemma PP2. Fix $\mathcal{A} \subset \mathcal{M}$, with $\mathcal{A} \neq \mathcal{M}$. Assume that the Strong Main LEMMA holds for each $\overline{\mathcal{A}}<\mathcal{A}$. Then the WEAK MAIN LEMMA holds for $\mathcal{A}$.

Lemma PP3. Fix $\mathcal{A} \subseteq \mathcal{M}$, and assume that the WEAK MAIN LEMMA holds for all $\overline{\mathcal{A}} \leq \mathcal{A}$. Then the StRong MAIN LEMma holds for $\mathcal{A}$.

Once we have established these three Lemmas, the two MAIN LEMMAS must hold for all $\mathcal{A}$, by induction on $\mathcal{A}$. Taking $\mathcal{A}$ to be the empty set in (say) the WEAK MAIN LEMMA, we see that hypotheses (WL1,2,3) hold vacuously; hence we obtain the following result.

Local Theorem 1. Given $m, n \geq 1$, there exist $A, c^{\prime}>0$, depending only on $m$ and $n$, for which the following holds.

Let $E \subset \mathbb{R}^{n}$ be finite, and let $\sigma: E \longrightarrow(0, \infty)$ be given. Let $y^{0} \in \mathbb{R}^{n}$. Then there exists a linear operator $\mathcal{E}: C^{m}(E, \sigma) \longrightarrow C^{m}\left(\mathbb{R}^{n}\right)$, with norm at most $A$, and satisfying

$$
|\mathcal{E} f(x)-f(x)| \leq A\|f\|_{C^{m}(E, \sigma)} \cdot \sigma(x)
$$

for all $f \in C^{m}(E, \sigma)$ and all $x \in E \cap B\left(y^{0}, c^{\prime}\right)$.

We will then relax the hypothesis $\sigma: E \rightarrow(0, \infty)$ to $\sigma: E \rightarrow[0, \infty)$, and next deduce Theorem 1 by using an obvious partition of unity. Finally, we deduce Theorem 2 from Theorem 1. These arguments are given in sections $14-17$. 


\section{Starting the Main Induction}

In this section, we prove Lemma PP1. That is, we prove the two MAIN LEMMAS for $\mathcal{A}=\mathcal{M}$. We simply take $\mathcal{E}=0$, and assume either (WL1,2,3) or $(\mathbf{S L 1}, 2,3)$, for our given $E, \sigma$.

Suppose $\|f\|_{C^{m}(E, \sigma)} \leq 1$. Then there exists $F \in C^{m}\left(\mathbb{R}^{n}\right)$ with

$$
\|F\|_{C^{m}\left(\mathbb{R}^{n}\right)} \leq 2 \text { and }|F(x)-f(x)| \leq 2 \sigma(x) \text { on } E .
$$

Hence, $|f(x)| \leq 2+2 \sigma(x)$ on $E$. On the other hand, the proof of (6.2) in Section 6 of [8] applies here, and shows that

$$
\sigma(x) \geq \frac{1}{2 C} \quad \text { for all } x \in E \cap B\left(y^{0}, c^{\prime}\right)
$$

with $C$ as in $(\mathbf{W L 1}, \mathbf{2}, \mathbf{3})$ or $(\mathbf{S L} \mathbf{1}, \mathbf{2}, \mathbf{3})$, and with $c^{\prime}$ determined by $C, m, n$. Consequently,

$$
|\mathcal{E} f(x)-f(x)|=|f(x)| \leq(4 C+2) \cdot \sigma(x) \text { for all } x \text { in } E \cap B\left(y^{0}, c^{\prime}\right) .
$$

This holds provided $\|f\|_{C^{m}(E, \sigma)} \leq 1$. The conclusions of the two MAIN LEMMAS are now obvious.

\section{Non-Monotonic Sets}

In this section, we prove Lemma PP2 for non-monotonic $\mathcal{A}$.

Lemma NMS. Fix a non-monotonic set $\mathcal{A} \subset \mathcal{M}$, and assume that the Strong main lemma holds for all $\overline{\mathcal{A}}<\mathcal{A}$. Then the Weak main Lemma holds for $\mathcal{A}$.

Proof. Let $E, \sigma$ satisfy $(\mathbf{W L 1}, 2,3)$ for $\mathcal{A}$. Since $\mathcal{A}$ is not monotonic, there exist multi-indices $\bar{\alpha}, \bar{\gamma}$, with $\bar{\alpha} \in \mathcal{A}, \bar{\alpha}+\bar{\gamma} \in \mathcal{M} \backslash \mathcal{A}$. We set $\overline{\mathcal{A}}=$ $\mathcal{A} \cup\{\bar{\alpha}+\bar{\gamma}\}$. As in the proof of Lemma 7.1 in [8], we see that $\overline{\mathcal{A}}<\mathcal{A}$, and that the hypotheses $(\mathrm{SL} \mathbf{1}, \mathbf{2}, \mathbf{3})$ of the STRONG MAIn LEMMA hold for $\overline{\mathcal{A}}$, with constants depending only on $C, m, n$ in $(\mathbf{W L} 1,2,3)$ for $\mathcal{A}$.

Applying the STROng MAIN LEMma for $\overline{\mathcal{A}}$, we obtain a linear operator $\mathcal{E}: C^{m}(E, \sigma) \longrightarrow C^{m}\left(\mathbb{R}^{n}\right)$, satisfying $(\mathbf{S L} 4,5)$, with constants $C^{\prime}, c^{\prime}$ depending only on $C, m, n$ in $(\mathbf{W L} \mathbf{1}, \mathbf{2}, \mathbf{3})$ for $\mathcal{A}$. However, $(\mathbf{S L 4}, \mathbf{5})$ are the same as the conclusions $(\mathbf{W L} \mathbf{4}, \mathbf{5})$ of the WEAK MAIN LEMMA for $\mathcal{A}$. The proof of Lemma NMS is complete. 


\section{A Consequence of the Main Inductive Assumption}

In this section, we establish the following result.

Lemma CMIA. Fix $\mathcal{A} \subset \mathcal{M}$, and assume that the STRONG MAIN LEMmA holds, for all $\overline{\mathcal{A}}<\mathcal{A}$. Then there exists $k_{\mathrm{old}}^{\#} \geq k_{s w}^{\#}(m, n)$, depending only on $m$ and $n$, for which the following holds.

Let $A>0$ be given, let $Q \in \mathbb{R}^{n}$ be a cube, $\hat{E} \subset \mathbb{R}^{n}$ a finite set, $\sigma: \hat{E} \longrightarrow(0, \infty)$ a function. Suppose that, for each $y \in Q^{* *}$, we are given a set $\overline{\mathcal{A}}^{y}<\mathcal{A}$ and a family of polynomials $\bar{P}_{\alpha}^{y} \in \mathcal{P}$, indexed by $\alpha \in \overline{\mathcal{A}}^{y}$. Assume that the following conditions are satisfied:

(G1) $\partial^{\beta} \bar{P}_{\alpha}^{y}(y)=\delta_{\beta \alpha}$ for all $\beta, \alpha \in \overline{\mathcal{A}}^{y}, y \in Q^{* *}$

(G2) $\left|\partial^{\beta} \bar{P}_{\alpha}^{y}(y)\right| \leq A \delta_{Q}^{|\alpha|-|\beta|}$ for all $\alpha \in \overline{\mathcal{A}}^{y}, \beta \geq \alpha, y \in Q^{* *}$.

(G3) Given $S \subset \hat{E}$ with $\#(S) \leq k_{\text {old }}^{\#}$, and given $y \in Q^{* *}$ and $\alpha \in \overline{\mathcal{A}}^{y}$, there exists $\varphi_{\alpha}^{S} \in C^{m}\left(\mathbb{R}^{n}\right)$, with

(a) $\left\|\partial^{m} \varphi_{\alpha}^{S}\right\|_{C^{0}\left(\mathbb{R}^{n}\right)} \leq A \cdot \delta_{Q}^{|\alpha|-m}$

(b) $\left|\varphi_{\alpha}^{S}(x)\right| \leq A \cdot \delta_{Q}^{|\alpha|-m} \cdot \sigma(x)$ for all $x \in S$,

(c) $J_{y}\left(\varphi_{\alpha}^{S}\right)=\bar{P}_{\alpha}^{y}$

Then there exists $\mathcal{E}: C^{m}\left(\hat{E}, \sigma ; \delta_{Q}\right) \longrightarrow C^{m}\left(\mathbb{R}^{n} ; \delta_{Q}\right)$, with the following properties:

(G4) $\mathcal{E}$ has norm at most $A^{\prime}$;

(G5) $|\mathcal{E} f(x)-f(x)| \leq A^{\prime} \delta_{Q}^{-m}\|f\|_{C^{m}\left(\hat{E}, \sigma ; \delta_{Q}\right)} \cdot \sigma(x)$ for all $f \in C^{m}\left(\hat{E}, \sigma ; \delta_{Q}\right)$ and all $x \in \hat{E} \cap Q^{*}$.

Here, $A^{\prime}$ depends only on $A, m, n$.

Proof. As in Section 8 of [8], a rescaling reduces matters to the case $\delta_{Q}=1$.

Let $\delta_{Q}=1$, and assume $(\mathbf{G} \mathbf{1}, \mathbf{2}, \mathbf{3})$. For each $y \in Q^{* *}$, the hypotheses (SL1,2,3) of the Strong MAIN LEMma for $\overline{\mathcal{A}}^{y}$ hold, with $\hat{E}, \sigma, y$, $\bar{P}_{\alpha}^{y}\left(\alpha \in \overline{\mathcal{A}}^{y}\right), A$ in place of $E, \sigma, y^{0}, P_{\alpha}(\alpha \in \mathcal{A}), C$ in $(\mathbf{S L} 1,2,3)$.

In fact, $(\mathbf{S L 1}, \mathbf{2}, \mathbf{3})$ are immediate from $(\mathbf{G} \mathbf{1}, \mathbf{2 , 3})$, provided we take $k_{\text {old }}^{\#}$ to be the max of $k_{s w}^{\#}(m, n)$ and the constants $k^{\#}$ appearing in the strong main lemma for all $\overline{\mathcal{A}}<\mathcal{A}$. 
Hence, the STRONG MAIN LEMma for $\overline{\mathcal{A}}^{y}$ produces an operator $\mathcal{E}_{y}$ : $C^{m}(\hat{E}, \sigma) \longrightarrow C^{m}\left(\mathbb{R}^{n}\right)$ satisfying

$$
\left\|\mathcal{E}_{y}\right\| \leq A^{\prime}
$$

and

$$
\left|\mathcal{E}_{y} f(x)-f(x)\right| \leq A^{\prime} \cdot\|f\|_{C^{m}(\hat{E}, \sigma)} \cdot \sigma(x)
$$

for all $f \in C^{m}(\hat{E}, \sigma), x \in \hat{E} \cap B\left(y, c^{\prime}\right), y \in Q^{* *}$.

Here, $A^{\prime}$ and $c^{\prime}$ are determined by $A, m, n$ in $(\mathbf{G} \mathbf{1}, \mathbf{2}, \mathbf{3})$.

Given $f \in C^{m}(\hat{E}, \sigma)$, we set $F^{y}=\mathcal{E}_{y} f$ for each $y \in Q^{* *}$. We then define $F$ from the $F^{y}$ as in (8.1)-(8.9) in [8]. Since $F \in C^{m}\left(\mathbb{R}^{n}\right)$ depends linearly on the $F^{y}$, which depend linearly on $f$, the map $\mathcal{E}: f \longrightarrow F$ is a linear operator from $C^{m}(\hat{E}, \sigma)$ to $C^{m}\left(\mathbb{R}^{n}\right)$. Moreover, if

$$
\|f\|_{C^{m}(\hat{E}, \sigma)} \leq 1
$$

then (8.1) and (8.2) show that

$$
\left\|F^{y}\right\|_{C^{m}\left(\mathbb{R}^{n}\right)} \leq A^{\prime}
$$

and

$$
\left|F^{y}(x)-f(x)\right| \leq A^{\prime} \cdot \sigma(x)
$$

for all $x \in \hat{E} \cap B\left(y, c^{\prime}\right)$.

Hence, the proof of (8.8) and (8.9) in [8] goes through here, and we have

$$
\|F\|_{C^{m}\left(\mathbb{R}^{n}\right)} \leq C^{\prime \prime}
$$

with $C^{\prime \prime}$ determined by $A, m, n$; and

$$
|F(x)-f(x)| \leq A^{\prime} \cdot \sigma(x)
$$

for all $x \in \hat{E} \cap Q^{*}$. That is,

$$
\|\mathcal{E} f\|_{C^{m}\left(\mathbb{R}^{n}\right)} \leq C^{\prime \prime} \text { if }\|f\|_{C^{m}(\hat{E}, \sigma)} \leq 1
$$

and

$$
|\mathcal{E} f(x)-f(x)| \leq A^{\prime} \cdot \sigma(x) \text { for all } x \in \hat{E} \cap Q^{*},
$$

provided $\|f\|_{C^{m}(\hat{E}, \sigma)} \leq 1$.

From (8.3) and (8.4) we obtain trivially the desired conclusions $(\mathbf{G} 4,5)$ in the case $\delta_{Q}=1$. The proof of the Lemma is complete. 


\section{Set-up for the Main Induction}

In this section, we give the set-up for the proof of Lemma PP2 in the monotonic case. We fix $m, n \geq 1$ and $\mathcal{A} \subset \mathcal{M}$. We let $k^{\#}$ be a large enough integer determined by $m$ and $n$, to be picked later. We suppose we are given $E \subset \mathbb{R}^{n}$ finite, $\sigma: E \longrightarrow(0, \infty), y^{0} \in \mathbb{R}^{n}, P_{\alpha} \in \mathcal{P}$ indexed by $\alpha \in \mathcal{A}$. In addition, we suppose we are given a positive number $a_{1}$. We fix $k^{\#}, E, \sigma, y^{0},\left(P_{\alpha}\right)_{\alpha \in \mathcal{A}}, a_{1}$ until the end of Section 12. We make the following assumptions.

(SU0) $\mathcal{A}$ is monotonic, and $\mathcal{A} \neq \mathcal{M}$.

(SU1) The Strong MAin LEmma holds for all $\overline{\mathcal{A}}<\mathcal{A}$.

(SU2) $\partial^{\beta} P_{\alpha}\left(y^{0}\right)=\delta_{\beta \alpha}$ for all $\beta, \alpha \in \mathcal{A}$.

(SU3) $\left|\partial^{\beta} P_{\alpha}\left(y^{0}\right)-\delta_{\beta \alpha}\right| \leq a_{1}$ for all $\alpha \in \mathcal{A}, \beta \in \mathcal{M}$.

(SU4) $a_{1}$ is less than a small enough constant determined by $m$ and $n$.

(SU5) Given $S \subset E$ with $\#(S) \leq k^{\#}$, and given $\alpha \in \mathcal{A}$, there exists $\varphi_{\alpha}^{S} \in$ $C^{m}\left(\mathbb{R}^{n}\right)$, with

(a) $\left\|\partial^{m} \varphi_{\alpha}^{S}\right\|_{C^{0}\left(\mathbb{R}^{n}\right)} \leq a_{1}$,

(b) $\left|\varphi_{\alpha}^{S}(x)\right| \leq \sigma(x)$ for all $x \in S$,

(c) $J_{y^{0}}\left(\varphi_{\alpha}^{S}\right)=P_{\alpha}$.

Most of the effort of this paper goes into proving the following result.

Lemma SU.I. Assume (SU0-SU5). Then there exists a linear operator $\mathcal{E}: C^{m}(E, \sigma) \longrightarrow C^{m}\left(\mathbb{R}^{n}\right)$, satisfying

(a) $\|\mathcal{E}\| \leq A$, and

(b) $|\mathcal{E} f(x)-f(x)| \leq A\|f\|_{C^{m}(E, \sigma)} \cdot \sigma(x)$ for all $f \in C^{m}(E, \sigma)$ and all $x \in E \cap B\left(y^{0}, a\right)$.

Here, $A$ and $a$ are determined by $a_{1}, m, n$.

Note that $a_{1}$ is not assumed to depend only on $m$ and $n$, and that the constant $C$ in (WL3) (b) has in effect been set equal to 1 in (SU5)(b).

The following result is trivial. (Compare with Lemma 9.2 in [8].)

Lemma SU.II. Lemma SU.I implies Lemma PP2. 


\section{Applying Lemmas on Convex Sets}

We place ourselves in the setting of Section 9, and we assume (SU0-SU5). Recall that we have fixed $E \subset \mathbb{R}^{n}$ finite, and $\sigma: E \longrightarrow(0, \infty)$.

For $y \in \mathbb{R}^{n}$ and $S \subset E$, we set

(10.1) $\Gamma(y, S)=\left\{J_{y}(\varphi):\|\varphi\|_{C^{m}\left(\mathbb{R}^{n}\right)} \leq 1\right.$, and $|\varphi(x)| \leq \sigma(x)$ on $\left.S\right\} \subseteq \mathcal{P}$.

Each $\Gamma(y, S)$ is bounded, convex, symmetric, and contains 0 as an interior point. Moreover, there are only finitely many subsets $S \subset E$. Hence, we may apply the LEMMA ON CONVEx SETs to the closures of the $\Gamma(y, S)$ for any fixed $y$, and $S \subset E$ arbitrary, subject to $\#(S) \leq k^{\#}$. Thus, we obtain subsets $S_{1}, \ldots, S_{D \cdot(D+1)} \subset E$, with $\#\left(S_{i}\right) \leq k^{\#}$ for each $i$, and satisfying the following inclusion:

$$
\bigcap_{i=1}^{D(D+1)} \Gamma\left(y, S_{i}\right) \subset C_{D} \cdot \bigcap_{\substack{S \subset E \\ \#(S) \leq k}} \text { Closure }(\Gamma(y, S)) .
$$

Moreover, Closure $(\Gamma(y, S)) \subset 2 \Gamma(y, S)$, since 0 is an interior point of the convex set $\Gamma(y, S)$. Hence, with $C_{D}^{\prime}=2 C_{D}$, we have

$$
\bigcap_{i=1}^{D \cdot(D+1)} \Gamma\left(y, S_{i}\right) \subset C_{D}^{\prime} \cdot \bigcap_{\substack{S \subset E \\ \#(S) \leq k \#}} \Gamma(y, S) .
$$

Here, $C_{D}^{\prime}$ depends only on $D$, and of course the $S_{i}$ depend on $y$. Let

$$
S^{y}=S_{1} \cup \cdots \cup S_{D(D+1)} .
$$

Then, obviously,

$$
\begin{aligned}
S^{y} & \subset E \\
\#\left(S^{y}\right) & \leq D \cdot(D+1) \cdot k^{\#},
\end{aligned}
$$

and $\Gamma\left(y, S^{y}\right) \subset \Gamma\left(y, S_{i}\right)$ for $i=1, \ldots, D(D+1)$, so that (10.2) implies

$$
\Gamma\left(y, S^{y}\right) \subset C_{D}^{\prime} \cdot \Gamma(y, S) \text { for all } S \subset E \text { with } \#(S) \leq k^{\#} .
$$

Next, using $S^{y}$, we introduce a linear operator

$$
T^{y}: C^{m}(E, \sigma) \longrightarrow \mathcal{P} .
$$

We proceed as follows. Let $S^{y} \cup\{y\}=\left\{x_{1}, \ldots, x_{N}\right\}$, with $x_{N}=y$. We introduce the vector space $\mathcal{P}^{N}$ of all

$$
\vec{P}=\left(P_{\mu}\right)_{1 \leq \mu \leq N} \quad \text { with each } P_{\mu} \in \mathcal{P} \text {. }
$$


Given a function $f \in C^{m}(E, \sigma)$, we define a quadratic function $\mathcal{Q}_{f}^{y}$ on $\mathcal{P}^{N}$, by setting

$$
\begin{aligned}
\mathcal{Q}_{f}^{y}(\vec{P})= & \sum_{\mu=1}^{N} \sum_{|\beta| \leq m-1}\left|\partial^{\beta} P_{\mu}\left(x_{\mu}\right)\right|^{2}+\sum_{\mu \neq \nu} \sum_{|\beta| \leq m-1} \frac{\left|\partial^{\beta}\left(P_{\mu}-P_{\nu}\right)\left(x_{\mu}\right)\right|^{2}}{\left|x_{\mu}-x_{\nu}\right|^{2 \cdot(m-|\beta|)}} \\
& +\sum_{\mu=1}^{N} 1_{x_{\mu} \in E} \cdot \frac{\left|P_{\mu}\left(x_{\mu}\right)-f\left(x_{\mu}\right)\right|^{2}}{\left(\sigma\left(x_{\mu}\right)\right)^{2}}
\end{aligned}
$$

for $\vec{P}$ as in (10.8). Here, the characteristic function $1_{x_{\mu} \in E}$ enters, since we don't know whether $y$ belongs to $E$.

The quadratic function $\mathcal{Q}_{f}^{y}$ contains $0^{\text {th }}, 1^{\text {st }}$, and $2^{\text {nd }}$ degree terms in $\vec{P}$. The sum of the second-degree terms is a strictly positive-definite quadratic form, independent of $f$. Also, the first degree terms are linear in $f$. It follows that $\vec{P} \mapsto \mathcal{Q}_{f}^{y}(\vec{P})$ achieves a minimum at a point $\vec{P}(f, y) \in \mathcal{P}^{N}$ that depends linearly on $f$ for fixed $y$. The components of $\vec{P}(f, y)$ may be denoted by $P_{\mu}(f, y) \in \mathcal{P}$, for $\mu=1, \ldots, N$. We define $T^{y}$ in (10.7) by setting

$$
T^{y} f=P_{N}(f, y) \text {. }
$$

Thus, $T^{y}$ is a linear operator from $C^{m}(E, \sigma)$ to $\mathcal{P}$. Note that

(10.11) $T^{y} f$ depends only on $\left.f\right|_{S^{y} \cup\{y\}}$ if $y \in E$, and only on $\left.f\right|_{S^{y}}$ if $y \notin E$.

Next, we prove the following result.

Lemma 1. Given $y \in \mathbb{R}^{n}$ and $f \in C^{m}(E, \sigma)$, there exists $\tilde{F} \in C^{m}\left(\mathbb{R}^{n}\right)$ with

$$
\begin{aligned}
\|\tilde{F}\|_{C^{m}\left(\mathbb{R}^{n}\right)} & \leq C\|f\|_{C^{m}(E, \sigma)}, \\
|\tilde{F}(x)-f(x)| & \leq C \cdot \sigma(x) \cdot\|f\|_{C^{m}(E, \sigma)} \text { for all } x \in S^{y}, \\
J_{y}(\tilde{F}) & =T^{y} f .
\end{aligned}
$$

Here, $C$ depends only on $m$ and $n$.

Proof. Throughout the proof, let $C$ denote a constant determined by $m$ and $n$. Without loss of generality, we may suppose that

$$
\|f\|_{C^{m}(E, \sigma)}=1 .
$$

By definition of the norm in (10.15), there exists $F \in C^{m}\left(\mathbb{R}^{n}\right)$, with

$$
\begin{aligned}
\|F\|_{C^{m}\left(\mathbb{R}^{n}\right)} & \leq 2, \text { and } \\
|F(x)-f(x)| & \leq 2 \sigma(x) \text { for all } x \in E .
\end{aligned}
$$


Define $\vec{P}=\left(P_{\mu}\right)_{1 \leq \mu \leq N} \in \mathcal{P}^{N}$ by setting $P_{\mu}=J_{x_{\mu}}(F)$. Thus, (10.16) and (10.17) imply the following estimates:

$$
\begin{aligned}
\left|\partial^{\beta} P_{\mu}\left(x_{\mu}\right)\right| & \leq 2 \text { for } 1 \leq \mu \leq N,|\beta| \leq m-1 . \\
\left|\partial^{\beta}\left(P_{\mu}-P_{\nu}\right)\left(x_{\mu}\right)\right| & \leq C\left|x_{\mu}-x_{\nu}\right|^{m-|\beta|} \quad \text { for } \mu \neq \nu,|\beta| \leq m-1 . \\
\left|P_{\mu}\left(x_{\mu}\right)-f\left(x_{\mu}\right)\right| & \leq 2 \sigma\left(x_{\mu}\right) \quad \text { if } x_{\mu} \in E .
\end{aligned}
$$

Hence, for this $\vec{P}$, each summand in (10.9) is at most $C$. Moreover, (10.5) shows that the number of summands in (10.9) is at most $C$.

Consequently, $\mathcal{Q}_{f}^{y}(\vec{P}) \leq C$. Since $\vec{P}(f, y)$ was picked to minimize $\mathcal{Q}_{f}^{y}$, we conclude that $\mathcal{Q}_{f}^{y}(\vec{P}(f, y)) \leq C$. In particular, we have

$$
\left|\partial^{\beta}\left[P_{\mu}(f, y)\right]\left(x_{\mu}\right)\right| \leq C
$$

for $1 \leq \mu \leq N,|\beta| \leq m-1$,

$$
\left|\partial^{\beta}\left[P_{\mu}(f, y)-P_{\nu}(f, y)\right]\left(x_{\mu}\right)\right| \leq C \cdot\left|x_{\mu}-x_{\nu}\right|^{m-|\beta|}
$$

for $\mu \neq \nu,|\beta| \leq m-1$ and

$$
\left|\left[P_{\mu}(f, y)\right]\left(x_{\mu}\right)-f\left(x_{\mu}\right)\right| \leq C \sigma\left(x_{\mu}\right)
$$

if $x_{\mu} \in E$.

By the standard Whitney extension theorem (see [11] or [15]), (10.18) and (10.19), there exists a function $\tilde{F} \in C^{m}\left(\mathbb{R}^{n}\right)$, with

$$
\|\tilde{F}\|_{C^{m}\left(\mathbb{R}^{n}\right)} \leq C
$$

and

$$
J_{x_{\mu}}(\tilde{F})=P_{\mu}(f, y) \quad \text { for each } \mu=1, \ldots N .
$$

By $(10.20),(10.22)$ and the definition of $x_{1}, \ldots, x_{N}$, we have

$$
|\tilde{F}(x)-f(x)| \leq C \sigma(x) \text { for all } x \in S^{y} .
$$

Moreover, (10.10) and (10.22) yield

$$
J_{y}(\tilde{F})=T^{y} f
$$

since $x_{N}=y$.

Under our assumption (10.15), results (10.21), (10.23) and (10.24) are precisely the conclusions (10.12), (10.13) and (10.14) of Lemma 1. The proof of the lemma is complete. 
For $y \in \mathbb{R}^{n}, f \in C^{m}(E, \sigma), M>0$, we define, as in [8], $\mathcal{K}_{f}\left(y, k^{\#}, M\right)$ as the set of $P \in \mathcal{P}$ such that, given $S \subset E$ with $\#(S) \leq k^{\#}$, there exists $F^{S} \in C^{m}\left(\mathbb{R}^{n}\right)$ with $\left\|F^{S}\right\|_{C^{m}\left(\mathbb{R}^{n}\right)} \leq M,\left|F^{S}(x)-f(x)\right| \leq M \cdot \sigma(x)$ for all $x \in S$ and $J_{y}\left(F^{S}\right)=P$.

Lemma 2. Given $y \in \mathbb{R}^{n}$ and $f \in C^{m}(E, \sigma)$ with $\|f\|_{C^{m}(E, \sigma)} \leq 1$, we have

$$
T^{y} f \in \mathcal{K}_{f}\left(y, k^{\#}, C\right)
$$

for a large enough $C$ determined by $m$ and $n$.

Proof. Let $\tilde{F}$ be as in Lemma 1. Since $\|f\|_{C^{m}(E, \sigma)} \leq 1$, we have

$$
\begin{aligned}
\|\tilde{F}\|_{C^{m}\left(\mathbb{R}^{n}\right)} & \leq C, \\
|\tilde{F}(x)-f(x)| & \leq C \cdot \sigma(x) \text { on } S^{y}, \text { and } \\
J_{y}(\tilde{F}) & =T^{y} f
\end{aligned}
$$

throughout the proof of Lemma $2, C, C^{\prime}$, c, etc. denote constants determined by $m$ and $n$.

Also, since $\|f\|_{C^{m}(E, \sigma)} \leq 1$, there exists $F \in C^{m}\left(\mathbb{R}^{n}\right)$, with

$$
\begin{aligned}
\|F\|_{C^{m}\left(\mathbb{R}^{n}\right)} & \leq C, \text { and } \\
|F(x)-f(x)| & \leq C \cdot \sigma(x) \text { on } E .
\end{aligned}
$$

From (10.25)-(10.29), and (10.4), we see that

$$
\begin{aligned}
\|F-\tilde{F}\|_{C^{m}\left(\mathbb{R}^{n}\right)} & \leq C, \\
|(F-\tilde{F})(x)| & \leq C \cdot \sigma(x) \text { on } S^{y}, \\
J_{y}(F-\tilde{F}) & =J_{y}(F)-T^{y} f .
\end{aligned}
$$

Comparing these results with definition (10.1), we see that

$$
J_{y}(F)-T^{y} f \in C \cdot \Gamma\left(y, S^{y}\right) .
$$

Now let $S \subset E$, with $\#(S) \leq k^{\#}$. By (10.6) and (10.30), we have

$$
J_{y}(F)-T^{y} f \in C^{\prime} \cdot \Gamma(y, S) .
$$

This means that there exists $\varphi^{S} \in C^{m}\left(\mathbb{R}^{n}\right)$, with

$$
\begin{aligned}
J_{y}\left(\varphi^{S}\right) & =J_{y}(F)-T^{y} f, \\
\left\|\varphi^{S}\right\|_{C^{m}\left(\mathbb{R}^{n}\right)} & \leq C^{\prime}, \\
\left|\varphi^{S}(x)\right| & \leq C^{\prime} \cdot \sigma(x) \text { on } S .
\end{aligned}
$$


We set $F^{S}=F-\varphi^{S}$. Then (10.31) gives

$$
J_{y}\left(F^{S}\right)=T^{y} f
$$

and from (10.28) and (10.32) we see that

$$
\left\|F^{S}\right\|_{C^{m}\left(\mathbb{R}^{n}\right)} \leq C^{\prime \prime}
$$

Also, (10.29) and (10.33) yield

$$
\left|F^{S}(x)-f(x)\right| \leq C^{\prime \prime \prime} \cdot \sigma(x) \text { on } S .
$$

Thus, given $S \subset E$ with $\#(S) \leq k^{\#}$, there exists $F^{S} \in C^{m}\left(\mathbb{R}^{n}\right)$, satisfying (10.34), (10.35) and (10.36). Thus $T^{y} f \in \mathcal{K}_{f}\left(y, k^{\#}, C^{\prime \prime \prime \prime}\right)$, which is the conclusion of Lemma 2. The proof of the lemma is complete.

Lemma 2 above substitutes for Lemma 10.1 in [8]. The proofs of Lemmas 10.2, 10.3 and 10.4 in [8] remain valid here. In place of Lemma 10.5 in [8], we use the following result.

Lemma 3. Suppose

$$
k^{\#} \geq(D+1) \cdot k_{1}^{\#} \quad \text { and } \quad k_{1}^{\#} \geq 1 .
$$

Let $y \in B\left(y^{0}, a_{1}\right)$ be given. Then there exists a linear map

$$
T_{y}^{\#}: C^{m}(E, \sigma) \longrightarrow \mathcal{P}
$$

with the following property:

If $\|f\|_{C^{m}(E, \sigma)} \leq 1$, then $T_{y}^{\#} f \in \mathcal{K}_{f}^{\#}\left(y ; k_{1}^{\#}, C\right)$, with $C$ depending only on $m$ and $n$.

Recall that $\mathcal{K}_{f}^{\#}(y ; k, M)$ consists of those polynomials $P \in \mathcal{K}_{f}(y ; k, M)$ for which $\partial^{\beta} P(y)=0$ for all $\beta \in \mathcal{A}$.

Proof of Lemma 3. We follow the proof of Lemma 10.5 in [8].

By Lemma 10.3 in [8], there exist polynomials $P_{\alpha}^{y} \in \mathcal{P}(\alpha \in \mathcal{A})$, satisfying $(\mathbf{W L 1})^{y}, \ldots,(\mathbf{W L 3})^{y}$ in $[8]$. We define

$$
T_{y}^{\#} f=T^{y} f-\sum_{\alpha \in \mathcal{A}}\left(\partial^{\alpha}\left(T^{y} f\right)(y)\right) \cdot P_{\alpha}^{y} .
$$

As promised, $T_{y}^{\#}$ is a linear map from $C^{m}(E, \sigma)$ to $\mathcal{P}$. Moreover, if $\|f\|_{C^{m}(E, \sigma)} \leq 1$, then Lemma 2 above gives $T^{y} f \in \mathcal{K}_{f}\left(y ; k^{\#}, C\right)$. Therefore, the proof of Lemma 10.5 in [8] applies, with $P=T^{y} f$ and $\tilde{P}=T_{y}^{\#} f$. (The constant 2 in (10.27) in [8] has to be replaced by $C$, but that has no effect on the rest of the argument.) Thus, as in the proof of Lemma 10.5 in [8], we find that $\tilde{P} \in \mathcal{K}_{f}^{\#}\left(y ; k_{1}^{\#}, C^{\prime \prime}\right)$. This is the desired property of $T_{y}^{\#} f$. The proof of Lemma 3 is complete. 


\section{Good News}

Again, we place ourselves in the setting of Sect. 9 and we assume SU0-SU5.

We define the cube $Q^{\circ}$ and its Calderón-Zygmund decomposition as in Section 11 of [8]. The good news is that all the arguments in Sections 11-14 of [8] work here as well. In particular, we have the crucial Lemma 14.3 from [8], which we restate here in a slightly weaker form than in [8].

Lemma GN. Let $y \in Q^{* *}$ and $y^{\prime} \in\left(Q^{\prime}\right)^{* *}$, where $Q$ and $Q^{\prime}$ are $C Z$ cubes. Let $f \in C^{m}(E, \sigma)$, and let $P \in \mathcal{K}_{f}^{\#}\left(y ; k_{A}^{\#}, C\right)$ and $P^{\prime} \in \mathcal{K}_{f}^{\#}\left(y^{\prime} ; k_{A}^{\#}, C\right)$ be given, where $C$ depends only on $m, n$; and assume

$$
k^{\#} \geq(D+1) \cdot k_{A}^{\#} \quad \text { and } \quad k_{A}^{\#} \geq(D+1)^{2} \cdot k_{\text {old }}^{\#} .
$$

If the cubes $Q$ and $Q^{\prime}$ abut, then we have

$$
\left|\partial^{\beta}\left(P^{\prime}-P\right)\left(y^{\prime}\right)\right| \leq C^{\prime} \cdot\left(a_{1}\right)^{-(m+1)} \delta_{Q}^{m-|\beta|} \quad \text { for all } \beta \in \mathcal{M} .
$$

Here, $C^{\prime}$ depends only on $m$ and $n$.

\section{Proof of Lemmas SU.I and PP2}

In this section, we prove Lemma SU.I. By Lemma SU.II, this will prove Lemma PP2 as well. We place ourselves in the setting of Section 9, and assume (SU0-SU5). In particular,

$$
\begin{aligned}
& E \text { is a given, finite subset of } \mathbb{R}^{n}, \\
& \sigma: E \longrightarrow(0, \infty) \text { is given, and } \\
& \mathcal{A} \subset \mathcal{M} \text { is given. }
\end{aligned}
$$

We use the Calderón-Zygmund decomposition from Section 11 of [8]. Let $Q_{\nu}\left(1 \leq \nu \leq \nu_{\max }\right)$ be the $C Z$ cubes, and let $\delta_{\nu}=\delta_{Q_{\nu}}=$ diameter of $Q_{\nu}$, $y_{\nu}=$ center of $Q_{\nu}$. Recall that

$$
\delta_{\nu} \leq a_{1} \leq 1 \quad \text { for each } \nu \text {, thanks to (11.3) in [8]. }
$$

We take

$$
k^{\#}=(D+1)^{3} \cdot k_{\text {old }}^{\#} \text {. }
$$

Let $\tilde{\theta}_{\nu}\left(1 \leq \nu \leq \nu_{\max }\right)$ be a cut-off function, with the following properties.

$$
\begin{aligned}
& 0 \leq \tilde{\theta}_{\nu} \leq 1 \text { on } \mathbb{R}^{n}, \tilde{\theta}_{\nu}=1 \text { on } Q_{\nu}^{*}, \operatorname{supp} \tilde{\theta}_{\nu} \subset Q_{\nu}^{* *}, \\
& \left|\partial^{\beta} \tilde{\theta}_{\nu}\right| \leq C \cdot \delta_{\nu}^{-|\beta|} \text { for } \beta \in \mathcal{M} .
\end{aligned}
$$

Throughout this section, we write $c, C, C^{\prime}$, etc. to denote constants determined by $m$ and $n$. 
Fix $\nu\left(1 \leq \nu \leq \nu_{\max }\right)$. Recall that, since $Q_{\nu}$ is a $C Z$ cube, it is OK. (See section 11 of [8] for the notion of an OK cube.) Thus,

For each $y \in Q_{\nu}^{* *}$, we are given $\overline{\mathcal{A}}^{y}<\mathcal{A}$, and polynomials $\bar{P}_{\alpha}^{y} \in \mathcal{P}\left(\alpha \in \overline{\mathcal{A}}^{y}\right)$ satisfying (OK1), (OK2), (OK3) in [8].

The following result is straightforward.

Lemma 1. The hypotheses of Lemma CMIA in Section 8 hold here, with $A=\left(a_{1}\right)^{-(m+1)}$, for the set $E$, the function $\sigma$, the cube $Q_{\nu}$, the sets of multiindices $\mathcal{A}$ and $\overline{\mathcal{A}}^{y}\left(y \in Q_{\nu}^{* *}\right)$, and the polynomials $\bar{P}_{\alpha}^{y}\left(y \in Q_{\nu}^{* *}, \alpha \in \overline{\mathcal{A}}^{y}\right)$.

Proof. The hypotheses of Lemma CMIA are as follows:

- The Strong main Lemma holds for all $\overline{\mathcal{A}}<\mathcal{A}$. (That's just (SU1), which we are assuming here.)

- $E \subset \mathbb{R}^{n}$ is finite, and $\sigma: E \longrightarrow(0, \infty)$. (That's contained in (12.1) and (12.2).)

- For each $y \in Q_{\nu}^{* *}$, we are given $\overline{\mathcal{A}}^{y}<\mathcal{A}$ and $\bar{P}_{\alpha}^{y}\left(\alpha \in \overline{\mathcal{A}}^{y}\right)$. (That's immediate from (12.8).)

- Conditions (G1), (G2), (G3) hold, with $A=\left(a_{1}\right)^{-(m+1)}$. (That's immediate from (OK1), (OK2), (OK3) for $Q_{\nu}$; these conditions hold, thanks to (12.8).)

The proof of Lemma 1 is complete.

From Lemma 1 and Lemma CMIA, we obtain a linear operator

$$
\begin{aligned}
& \mathcal{E}_{\nu}: C^{m}\left(E, \sigma ; \delta_{\nu}\right) \longrightarrow C^{m}\left(\mathbb{R}^{n} ; \delta_{\nu}\right), \text { satisfying } \\
& \qquad\left\|\mathcal{E}_{\nu}\right\| \leq A^{\prime}, \text { and } \\
& {\left[\begin{array}{l}
\left|\mathcal{E}_{\nu} f(x)-f(x)\right| \leq A^{\prime} \cdot\|f\|_{C^{m}\left(E, \sigma ; \delta_{\nu}\right)} \cdot \delta_{\nu}^{-m} \cdot \sigma(x) \\
\text { for all } f \in C^{m}\left(E, \sigma ; \delta_{\nu}\right) \text { and all } x \in E \cap Q_{\nu}^{*} .
\end{array}\right]}
\end{aligned}
$$

Here, and throughout this section, $A^{\prime}, A^{\prime \prime}, A$, a, etc., denote constants determined by $a_{1}, m, n$.

Next, we bring in Lemma 3 from Section 10, applied with

$$
k_{1}^{\#}=(D+1)^{2} \cdot k_{\text {old }}^{\#}, \quad y=y_{\nu} .
$$

(Note that $y_{\nu} \in B\left(y^{0}, a_{1}\right)$ as required in Lemma 3, since $y_{\nu} \in Q_{\nu} \subseteq Q^{\circ} \subset$ $B\left(y^{0}, a_{1}\right)$.) Thus, we obtain a linear map

$$
T_{\nu}^{\#}: C^{m}(E, \sigma) \longrightarrow \mathcal{P}
$$

satisfying the following:

$$
\text { If }\|f\|_{C^{m}(E, \sigma)} \leq 1, \text { then } T_{\nu}^{\#} f \in \mathcal{K}_{f}^{\#}\left(y_{\nu} ;(D+1)^{2} \cdot k_{\text {old }}^{\#}, C\right) .
$$


Using the map $T_{\nu}^{\#}$ and the cut-off function $\tilde{\theta}_{\nu}$ (see (12.6), (12.7)), we define a linear map

$$
L_{\nu}: C^{m}(E, \sigma) \longrightarrow C^{m}\left(E, \sigma ; \delta_{\nu}\right)
$$

by setting

$$
\left(L_{\nu} f\right)(x)=\left(f(x)-\left(T_{\nu}^{\#} f\right)(x)\right) \cdot \tilde{\theta}_{\nu}(x) \text { for all } x \in \mathbb{R}^{n} .
$$

Lemma 2. The norm of $L_{\nu}$ from $C^{m}(E, \sigma)$ to $C^{m}\left(E, \sigma ; \delta_{\nu}\right)$, is at most $C^{\prime} \delta_{\nu}^{m}$. Proof. Suppose $\|f\|_{C^{m}(E, \sigma)} \leq 1$. Then $(12)$ and the definition of $\mathcal{K}_{f}^{\#}$ yield the following.

(12.15) Let $S \subset E$, with $\#(S) \leq k_{\text {old }}^{\#}$. Then there exists $F^{S} \in C^{m}\left(\mathbb{R}^{n}\right)$, with

$$
\begin{aligned}
\left\|F^{S}\right\|_{C^{m}\left(\mathbb{R}^{n}\right)} & \leq C, \\
\left|F^{S}(x)-f(x)\right| & \leq C \cdot \sigma(x) \text { on } S, \text { and } \\
J_{y_{\nu}}\left(F^{S}\right) & =T_{\nu}^{\#} f .
\end{aligned}
$$

From (12.16), (12.18) and Taylor's theorem, we have

$$
\left|\partial^{\beta}\left(F^{S}-T_{\nu}^{\#} f\right)\right| \leq C \delta_{\nu}^{m-|\beta|} \text { on } Q_{\nu}^{* *} \text {, for }|\beta| \leq m .
$$

Together with properties $(12.6),(12.7)$ of $\tilde{\theta}_{\nu}$, this implies that

$$
\left|\partial^{\beta}\left\{\tilde{\theta}_{\nu} \cdot\left(F^{S}-T_{\nu}^{\#} f\right)\right\}\right| \leq C \delta_{\nu}^{m-|\beta|} \text { on } \mathbb{R}^{n} \text {, for }|\beta| \leq m .
$$

On the other hand, (12.6), (12.14) and (12.17) show that

$$
\begin{aligned}
\left|\left\{\tilde{\theta}_{\nu} \cdot\left(F^{S}-T_{\nu}^{\#} f\right)\right\}(x)-L_{\nu} f(x)\right| & =\left|\tilde{\theta}_{\nu}(x) \cdot\left(F^{S}(x)-f(x)\right)\right| \\
& \leq\left|F^{S}(x)-f(x)\right| \leq C \cdot \sigma(x)
\end{aligned}
$$

for all $x \in S$. Together with (12.19), this shows the following:

(12.20) Given $S \subset E$ with $\#(S) \leq k_{\text {old }}^{\#}$, there exists $\tilde{F}^{S} \in C^{m}\left(\mathbb{R}^{n}\right)$, with

$$
\begin{aligned}
\left\|\partial^{\beta} \tilde{F}\right\|_{C^{0}\left(\mathbb{R}^{n}\right)} & \leq C \delta_{\nu}^{m-|\beta|} \text { for }|\beta| \leq m \text { and } \\
\left|\tilde{F}^{S}(x)-L_{\nu} f(x)\right| & \leq C \cdot \sigma(x) \text { for all } x \in S .
\end{aligned}
$$

Comparing (12.20) with the definition of the $C^{m}(E, \sigma ; \delta)$ norm, we learn that

(12.21) $\quad\left\|L_{\nu} f\right\|_{C^{m}\left(S,\left.\sigma\right|_{S} ; \delta_{\nu}\right)} \leq C \cdot \delta_{\nu}^{m}$ for all $S \subset E$ with $\#(S) \leq k_{\text {old }}^{\#}$.

We recall from Lemma CMIA that $k_{\text {old }}^{\#} \geq k_{s w}^{\#}(m, n)$. Consequently, (12.21) and the Corollary in section 2 together imply that

$$
\left\|L_{\nu} f\right\|_{C^{m}\left(E, \sigma ; \delta_{\nu}\right)} \leq C \cdot \delta_{\nu}^{m} .
$$

This holds whenever $\|f\|_{C^{m}(E, \sigma)} \leq 1$. The proof of Lemma 2 is complete. 
Next, we introduce a partition of unity on $Q^{0}$. We no longer fix $\nu$. For each $\nu\left(1 \leq \nu \leq \nu_{\max }\right)$, we introduce a cut-off function $\hat{\theta}_{\nu}$, satisfying

$$
0 \leq \hat{\theta}_{\nu} \leq 1 \text { on } \mathbb{R}^{n}, \hat{\theta}_{\nu}=1 \text { on } Q_{\nu}, \hat{\theta}_{\nu}(x)=0 \text { for } \operatorname{dist}\left(x, Q_{\nu}\right)>\hat{c} \delta_{\nu}
$$

and

$$
\left|\partial^{\beta} \hat{\theta}_{\nu}\right| \leq C \delta_{\nu}^{-|\beta|} \text { for }|\beta| \leq m
$$

Taking $\hat{c}$ small enough in (12.22), and recalling Lemma 11.2 in [8], we obtain the following.

(12.24) If $Q_{\mu}$ contains a point of $\operatorname{supp} \hat{\theta}_{\nu}$, then $Q_{\mu}$ and $Q_{\nu}$ coincide or abut.

Define $\theta_{\nu}=\hat{\theta}_{\nu} /\left(\sum_{\mu} \hat{\theta}_{\mu}\right)$ on $Q^{0}$. From (12.22), (12.23), (12.24), the Corollary to Lemma 11.1 in [8], and Lemma 11.2 in [8], we obtain:

$$
\begin{gathered}
\sum_{1 \leq \nu \leq \nu_{\max }} \theta_{\nu}=1 \text { on } Q^{0} . \\
0 \leq \theta_{\nu} \leq 1 \text { on } Q^{\circ} . \\
\left|\partial^{\beta} \theta_{\nu}\right| \leq C \delta_{\nu}^{-|\beta|} \text { for }|\beta| \leq m . \\
\theta_{\nu}=0 \text { outside } Q_{\nu}^{*} . \\
\text { If } x \in Q_{\mu} \text {, then } \theta_{\nu}=0 \text { in a neighborhood of } x, \\
\text { unless } Q_{\mu} \text { and } Q_{\nu} \text { coincide or abut. }
\end{gathered}
$$

Now we define

$$
\tilde{\mathcal{E}} f=\sum_{1 \leq \nu \leq \nu_{\max }} \theta_{\nu} \cdot\left[T_{\nu}^{\#} f+\mathcal{E}_{\nu}\left(L_{\nu} f\right)\right] \text { on } Q^{\circ} .
$$

Note that $\theta_{\nu}$ and $\tilde{\mathcal{E}} f$ are defined only on $Q^{\circ}$. Since $T_{\nu}^{\#}, \mathcal{E}_{\nu}$, and $L_{\nu}$ are linear, (12.30) shows that

$$
\tilde{\mathcal{E}} \text { is a linear map from } C^{m}(E, \sigma) \text { to } C^{m}\left(Q^{\circ}\right) .
$$

Suppose that $f$ is given with

$$
\|f\|_{C^{m}(E, \sigma)} \leq 1
$$

Then, for each $\nu$, we have

$$
\left\|L_{\nu} f\right\|_{C^{m}\left(E, \sigma ; \delta_{\nu}\right)} \leq C \delta_{\nu}^{m}
$$

by Lemma 2 . 
Hence, (12.9), (12.9a), (12.10) yield the estimates

$$
\left\|\mathcal{E}_{\nu}\left(L_{\nu} f\right)\right\|_{C^{m}\left(\mathbb{R}^{n} ; \delta_{\nu}\right)} \leq A^{\prime \prime} \cdot \delta_{\nu}^{m}
$$

(12.35) $\left|\mathcal{E}_{\nu}\left(L_{\nu} f\right)(x)-L_{\nu} f(x)\right| \leq A^{\prime \prime} \cdot \sigma(x)$ for all $x \in E \cap Q_{\nu}^{*}$.

For all $x \in E \cap Q^{\circ}$, we have

$$
\begin{aligned}
& \left|\theta_{\nu} \cdot\left[T_{\nu}^{\#} f+\mathcal{E}_{\nu}\left(L_{\nu} f\right)\right](x)-\theta_{\nu} \cdot f(x)\right|= \\
& =\theta_{\nu}(x) \cdot\left|T_{\nu}^{\#} f(x)+\left[\mathcal{E}_{\nu}\left(L_{\nu} f\right)(x)-L_{\nu} f(x)\right]+L_{\nu} f(x)-f(x)\right|= \\
& =\theta_{\nu}(x) \cdot \mid T_{\nu}^{\#} f(x)+\left[\mathcal{E}_{\nu}\left(L_{\nu} f\right)(x)-L_{\nu} f(x)\right]+ \\
& \quad+\tilde{\theta}_{\nu}(x) \cdot\left[f(x)-T_{\nu}^{\#} f(x)\right]-f(x) \mid \\
& =\theta_{\nu}(x) \cdot\left|T_{\nu}^{\#} f(x)+\left[\mathcal{E}_{\nu}\left(L_{\nu} f\right)(x)-L_{\nu} f(x)\right]+\left[f(x)-T_{\nu}^{\#} f(x)\right]-f(x)\right| \\
& \left.\quad \quad \quad \text { because } \tilde{\theta}_{\nu}=1 \text { on } \operatorname{supp} \theta_{\nu} ; \text { see }(12.6) \text { and }(12.28)\right) \\
& =\theta_{\nu}(x) \cdot\left|\mathcal{E}_{\nu}\left(L_{\nu} f\right)(x)-L_{\nu} f(x)\right| \leq \theta_{\nu}(x) \cdot A^{\prime \prime} \cdot \sigma(x)
\end{aligned}
$$

(thanks to (12.35) when $x \in Q_{\nu}^{*}$, and thanks to (12.28) when $x \notin Q_{\nu}^{*}$ ). Summing over $\nu$, and recalling (12.25) and (12.30), we find that

$$
|\tilde{\mathcal{E}} f(x)-f(x)| \leq A^{\prime \prime} \cdot \sigma(x) \text { for all } x \in E \cap Q^{\circ} .
$$

We prepare to estimate the derivatives of $\tilde{\mathcal{E}} f$. To do so, we note that (12.12) and (12.32) yield

$$
\left|\partial^{\beta}\left(T_{\nu}^{\#} f\right)\left(y_{\nu}\right)\right| \leq C \text { for }|\beta| \leq m-1,
$$

since we may take $S=$ empty set in the definition of $\mathcal{K}_{f}^{\#}\left(y_{\nu} ;(D+1)^{2} \cdot k_{\text {old }}^{\#}, C\right)$. For $|\beta|=m$, we have $\partial^{\beta}\left(T_{\nu}^{\#} f\right) \equiv 0$, since $T_{\nu}^{\#} f \in \mathcal{P}$. Thus,

$$
\left|\partial^{\beta}\left(T_{\nu}^{\#} f\right)\left(y_{\nu}\right)\right| \leq C \text { for }|\beta| \leq m .
$$

Since $T_{\nu}^{\#} f \in \mathcal{P},(12.37)$ and (12.4) show that

$$
\left|\partial^{\beta}\left(T_{\nu}^{\#} f\right)\right| \leq C \text { on } Q_{\nu}^{*} \text {, for }|\beta| \leq m .
$$

We need to compare $T_{\nu}^{\#} f$ with $T_{\mu}^{\#} f$ when $Q_{\nu}$ and $Q_{\mu}$ abut. From (12.12) and (12.32), we have

$$
\begin{aligned}
& T_{\nu}^{\#} f \in \mathcal{K}_{f}^{\#}\left(y_{\nu} ;(D+1)^{2} \cdot k_{\text {old }}^{\#}, C\right), \text { and } \\
& T_{\mu}^{\#} f \in \mathcal{K}_{f}^{\#}\left(y_{\mu} ;(D+1)^{2} \cdot k_{\text {old }}^{\#}, C\right) .
\end{aligned}
$$

Hence, we may apply Lemma GN (in section 11 ), with $k_{A}^{\#}=(D+1)^{2} \cdot k_{\text {old }}^{\#}$. (See (12.5).) Thus,

$$
\left|\partial^{\beta}\left(T_{\mu}^{\#} f-T_{\nu}^{\#} f\right)\left(y_{\mu}\right)\right| \leq A \cdot \delta_{\nu}^{m-|\beta|} \text { for }|\beta| \leq m-1 .
$$


Recalling Lemma 11.2 in [8], and recalling that $T_{\mu}^{\#} f, T_{\nu}^{\#} f \in \mathcal{P}$, we conclude that

$$
\left[\begin{array}{l}
\left|\partial^{\beta}\left(T_{\mu}^{\#} f-T_{\nu}^{\#} f\right)\right| \leq A^{\prime} \cdot \delta_{\mu}^{m-|\beta|} \text { on } Q_{\nu}^{*} \cup Q_{\mu}^{*} \text { for }|\beta| \leq m \\
\text { whenever } Q_{\mu} \text { and } Q_{\nu} \text { abut. }
\end{array}\right]
$$

We are almost ready to estimate the derivatives of $\tilde{\mathcal{E}} f$. It is convenient to set

$$
P_{\nu}=T_{\nu}^{\#} f, F_{\nu}=\mathcal{E}_{\nu}\left(L_{\nu} f\right), \tilde{F}=\tilde{\mathcal{E}} f .
$$

Thus, $P_{\nu} \in \mathcal{P}, F_{\nu} \in C^{m}\left(\mathbb{R}^{n}\right)$, and we have the following estimates:

- $\left|\partial^{\beta} P_{\nu}\right| \leq C$ on $Q_{\nu}^{*}$, for $|\beta| \leq m$. (See (12.38).)

- $\left|\partial^{\beta}\left(P_{\nu}-P_{\mu}\right)\right| \leq A^{\prime} \cdot \delta_{\mu}^{m-|\beta|}$ on $Q_{\mu}^{*} \cup Q_{\nu}^{*}$ for $|\beta| \leq m$, whenever $Q_{\mu}$ and $Q_{\nu}$ abut. (See (12.39).)

- $\left|\partial^{\beta} F_{\nu}\right| \leq A^{\prime \prime} \cdot \delta_{\nu}^{m-|\beta|}$ on $\mathbb{R}^{n}$, for $|\beta| \leq m$ (See (12.34).)

Moreover,

$$
\text { - } \tilde{F}=\sum_{1 \leq \nu \leq \nu_{\max }} \theta_{\nu} \cdot\left[P_{\nu}+F_{\nu}\right] \quad(\operatorname{See}(12.30) .)
$$

Thanks to the above bullets and properties (12.25)-(12.29) of the $\theta_{\nu}$ 's, the discussion in Section 15 of [8], starting at (15.32) and ending at (15.40) there, applies here as well. (The idea goes back to Whitney.) In particular, estimate (15.40) there yields here the estimate

$$
\left|\partial^{\beta}(\tilde{\mathcal{E}} f)(x)\right| \leq A^{\prime \prime \prime} \text { for all } x \in Q^{\circ},|\beta| \leq m .
$$

The extension operator has the good property that (12.36) and (12.40) hold whenever $f$ satisfies (12.32). However, $\tilde{\mathcal{E}} f$ is only defined on $Q^{\circ}$.

To remedy this, we pick a cut-off function $\theta^{\circ} \in C^{m}\left(\mathbb{R}^{n}\right)$, with

$$
\begin{aligned}
& \theta^{\circ}=1 \text { on } B\left(y^{0}, c^{\prime} a_{1}\right), \operatorname{supp} \theta^{\circ} \subset Q^{\circ}, 0 \leq \theta^{\circ} \leq 1 \text { on } \mathbb{R}^{n} \text {, and } \\
& \left|\partial^{\beta} \theta^{\circ}\right| \leq C a_{1}^{-|\beta|} \text { for }|\beta| \leq m .
\end{aligned}
$$

Setting $\mathcal{E} f=\theta \cdot(\tilde{\mathcal{E}} f)$, we obtain a linear operator

$$
\mathcal{E}: C^{m}(E, \sigma) \longrightarrow C^{m}\left(\mathbb{R}^{n}\right) .
$$

From (12.40) and the defining properties of $\theta^{\circ}$, we see that

$$
\|\mathcal{E} f\|_{C^{m}\left(\mathbb{R}^{n}\right)} \leq A_{4} \quad \text { if }\|f\|_{C^{m}(E, \sigma)} \leq 1 .
$$


From (12.36) and the defining properties of $\theta^{\circ}$, we see that

$$
|\mathcal{E} f(x)-f(x)| \leq A^{\prime \prime} \cdot \sigma(x) \text { for all } x \in E \cap B\left(y^{0}, c^{\prime} a_{1}\right),
$$

provided $\|f\|_{C^{m}(E, \sigma)} \leq 1$.

The conclusions (a) and (b) of Lemma SU.I are immediate from (12.42) and (12.43). (We may take $a=c^{\prime} a_{1}$.) The proofs of Lemmas SU.I and PP2 are complete.

\section{Proof of Lemma PP3}

In this section, we prove Lemma PP3. We fix $\mathcal{A} \subset \mathcal{M}$, and assume that the WEAK MAIN LEMma holds for all $\overline{\mathcal{A}} \leq \mathcal{A}$. We must show that the STRONG MAIN LEMMA holds for $\mathcal{A}$. We may assume that the WEAK MAIN LEMMA holds for all $\overline{\mathcal{A}} \leq \mathcal{A}$, with $k^{\#}$ and $a_{0}$ independent of $\overline{\mathcal{A}}$. (Although each $\overline{\mathcal{A}} \leq \mathcal{A}$ gives rise to its own $k^{\#}$ and $a_{0}$, we may simply use the maximum of all the $k^{\#}$, and the minimum of all the $a_{0}$, arising in the WEAK MAIN LEMMA for all $\overline{\mathcal{A}} \leq \mathcal{A}$.) Fix $k^{\#}$ and $a_{0}$ as in the WEAK MAIN LEMMA for $\overline{\mathcal{A}} \leq \mathcal{A}$.

Let $E, \sigma, y^{0}, P_{\alpha}(\alpha \in \mathcal{A})$ satisfy the hypotheses of the Strong MAIN LEMMA for $\mathcal{A}$. Without loss of generality, we may suppose

$$
y^{0}=0 \text {. }
$$

We want to find a linear operator $\mathcal{E}: C^{m}(E, \sigma) \rightarrow C^{m}\left(\mathbb{R}^{n}\right)$ satisfying $(\mathbf{S L 4 , 5})$.

In this section, we say that a constant is "controlled" if it is determined by $C, m, n$ in the hypotheses $(\mathbf{S L} \mathbf{1}, \mathbf{2}, \mathbf{3})$ of the Strong main lemma for $\mathcal{A}$. We write $c, C, C^{\prime}, C_{1}$, etc. to denote controlled constants. Also, we introduce a small constant $\bar{a}$ to be picked later. Initially, we do not assume that $\bar{a}$ is a controlled constant. We say that a constant is "weakly controlled" if it is determined by $\bar{a}$, together with $C, m, n$ in $(\mathbf{S L 1}, \mathbf{2}, \mathbf{3})$. We write $c(\bar{a})$, $C(\bar{a}), C^{\prime}(\bar{a})$, etc., to denote weakly controlled constants. Note that the constants $k^{\#}$ and $a_{0}$ are controlled. We assume that

$\bar{a}$ is less than a small enough controlled constant.

We proceed as in Sections 16 and 17 of [8]. Section 16 of [8] goes through unchanged here. We introduce the linear map

$$
T:\left(\hat{x}_{1}, \ldots, \hat{x}_{n}\right) \mapsto\left(\lambda_{1} \hat{x}_{1}, \ldots, \lambda_{n} \hat{x}_{n}\right),
$$

with $\lambda_{1}, \ldots, \lambda_{n}>0$ picked as in Section 17 of [8]. 
We define

$$
\hat{E}=T^{-1}(E), \hat{\sigma}=\sigma \circ T \text {. }
$$

As in Section 17 of [8], we may construct a set of multi-indices

$$
\overline{\mathcal{A}} \leq \mathcal{A}
$$

for which the following result is valid.

Lemma 1. (WL1), (WL2), (WL3) hold for the set $\hat{E}$, the function $\hat{\sigma}$, the set $\overline{\mathcal{A}}$ of multi-indices, the base point $y^{0}=0$, and for some family of polynomials $\bar{P}_{\bar{\alpha}}(\bar{\alpha} \in \overline{\mathcal{A}})$. Moreover, the constant called $C$ in hypothesis (WL3) for $\overline{\mathcal{A}}, \hat{E}, \hat{\sigma}, y^{0}=0,\left(\bar{P}_{\bar{\alpha}}\right)_{\bar{\alpha} \in \overline{\mathcal{A}}}$, is weakly controlled.

To prove Lemma 1, we just repeat the argument from (17.4a) through(17.27) of [8], omitting the discussion of $F^{S}$ and $\hat{F}^{\hat{S}}$.

Since we are assuming that the WEAK MAIN LEMma holds for all $\overline{\mathcal{A}} \leq \mathcal{A}$, we obtain from (13.5) and Lemma 1 that there exists a linear operator

$$
\begin{gathered}
\hat{\mathcal{E}}: C^{m}(\hat{E}, \hat{\sigma}) \longrightarrow C^{m}\left(\mathbb{R}^{n}\right) \text {, satisfying } \\
\qquad \hat{\mathcal{E}} \| \leq C_{1}(\bar{a}), \quad \text { and } \\
{\left[\begin{array}{c}
|\hat{\mathcal{E}} \hat{f}(\hat{x})-\hat{f}(\hat{x})| \leq C_{1}(\bar{a}) \cdot\|\hat{f}\|_{C^{m}(\hat{E}, \hat{\sigma})} \cdot \hat{\sigma}(\hat{x}) \\
\text { for all } \hat{f} \in C^{m}(\hat{E}, \hat{\sigma}), \text { and for all } \hat{x} \in \hat{E} \cap B\left(0, c_{1}(\bar{a})\right)
\end{array}\right]}
\end{gathered}
$$

Now, given $f \in C^{m}(E, \sigma)$, we define $\hat{f}=f \circ T$ on $\hat{E}$, then set

$$
\mathcal{E} f=(\hat{\mathcal{E}} \hat{f}) \circ T^{-1} .
$$

Thus, $\mathcal{E}$ is a linear operator from $C^{m}(E, \sigma)$ to $C^{m}\left(\mathbb{R}^{n}\right)$. Since $\lambda_{1}, \ldots, \lambda_{n}$ in (13.3) satisfy

$$
c(\bar{a}) \leq \lambda_{i} \leq 1 \quad(i=1, \ldots, n)
$$

(see [8] estimate (17.7)), the operator $f \mapsto \hat{f}$ has norm at most $C(\bar{a})$ as a map from $C^{m}(E, \sigma)$ to $C^{m}(\hat{E}, \hat{\sigma})$. Similarly, (13.9) shows that

$$
\|\mathcal{E} f\|_{C^{m}\left(\mathbb{R}^{n}\right)} \leq C(\bar{a}) \cdot\|\hat{\mathcal{E}} \hat{f}\|_{C^{m}\left(\mathbb{R}^{n}\right)} .
$$

Together with (13.7), this shows that the operator

$$
\mathcal{E}: C^{m}(E, \sigma) \longrightarrow C^{m}\left(\mathbb{R}^{n}\right) \quad \text { has norm at most } C_{2}(\bar{a}) \text {. }
$$


Also, from (13.8), (13.9), (13.10), we see that

$$
\left[\begin{array}{c}
|\mathcal{E} f(x)-f(x)| \leq C_{1}(\bar{a}) \cdot\|\hat{f}\|_{C^{m}(\hat{E}, \hat{\sigma})} \cdot \sigma(x) \\
\text { whenever } x \in E \text { and } T^{-1} x \in B\left(0, c_{1}(\bar{a})\right) .
\end{array}\right]
$$

Another application of (13.10) shows that

$$
\left[\begin{array}{c}
x \in B\left(0, c_{3}(\bar{a})\right) \text { implies } T^{-1} x \in B\left(0, c_{1}(\bar{a})\right), \\
\text { for a suitable weakly controlled constant } c_{3}(\bar{a}) .
\end{array}\right]
$$

Again using the fact that the operator $f \mapsto \hat{f}$ has norm at most $C(\bar{a})$ as a map from $C^{m}(E, \sigma)$ to $C^{m}(\hat{E}, \hat{\sigma})$, we derive from (13.12) and (13.13) the following conclusion.

$$
\left[\begin{array}{c}
|\mathcal{E} f(x)-f(x)| \leq C_{3}(\bar{a}) \cdot\|f\|_{C^{m}(E, \sigma)} \cdot \sigma(x), \\
\text { whenever } f \in C^{m}(E, \sigma) \text { and } x \in E \cap B\left(0, c_{3}(\bar{a})\right) .
\end{array}\right]
$$

Thus, if $\bar{a}$ satisfies (13.2), then the operator $\mathcal{E}$ satisfies (13.11) and (13.14). We now take $\bar{a}$ to be a controlled constant, small enough to satisfy (13.2). Then the constants $C_{2}(\bar{a}), C_{3}(\bar{a}), c_{3}(\bar{a})$ are determined entirely by $C, m, n$ in hypotheses $(\mathbf{S L 1}, \mathbf{2}, \mathbf{3})$. Hence, (13.11) and (13.14) are the desired properties $(\mathbf{S L 4 , 5})$ for the linear operator $\mathcal{E}$. Thus, the Strong MAIN LEMmA holds for $\overline{\mathcal{A}}$.

The proof of Lemma PP3 is complete.

\section{Proof of Theorem 1}

By now, we have proven Lemmas PP1, PP2 and PP3. Consequently, we have established the Local Theorem 1 in Section 5. To pass to Theorem 1, we first prove the following simple result.

Lemma 1. In the Local Theorem 1 , the hypothesis $\sigma: E \longrightarrow(0, \infty)$ may be relaxed to $\sigma: E \longrightarrow[0, \infty)$.

Proof. Let $E \subset \mathbb{R}^{n}$ be finite, let $\sigma: E \longrightarrow[0, \infty)$, and let $y^{0} \in \mathbb{R}^{n}$. Since $E$ is finite there exists a linear operator

$$
\mathcal{E}^{\text {trivial }}: C^{0}(E) \longrightarrow C^{m}\left(\mathbb{R}^{n}\right),
$$

with

$$
\mathcal{E}^{\text {trivial }} f(x)=f(x) \quad \text { for all } x \in E \text {. }
$$


We have

$$
\left\|\mathcal{E}^{\text {trivial }} f\right\|_{C^{m}\left(\mathbb{R}^{n}\right)} \leq \Gamma(E) \cdot\|f\|_{C^{0}(E)}
$$

for all $f$, with $\Gamma(E)$ a finite constant depending on $E$. We write

$$
E=E_{0} \cup E_{1},
$$

with

$$
E_{0}=\{x \in E: \sigma(x)=0\} \quad \text { and } \quad E_{1}=\{x \in E: \sigma(x)>0\} .
$$

For a small enough $\epsilon>0$ to be picked below, define

$$
\sigma_{\epsilon}(x)=\left\{\begin{array}{lll}
\sigma(x) & \text { if } & x \in E_{1} \\
\epsilon & \text { if } & x \in E_{0}
\end{array}\right.
$$

Thus, $\sigma_{\epsilon}: E \longrightarrow(0, \infty)$, so the Local Theorem 1 applies to $E, \sigma_{\epsilon}$. Let $\mathcal{E}$ be the operator provided by the Local Theorem 1 for $E, \sigma_{\epsilon}$. Note that

$$
\|f\|_{C^{m}\left(E, \sigma_{\epsilon}\right)} \leq\|f\|_{C^{m}(E, \sigma)} \text { for any } f \text {, simply because } \sigma_{\epsilon} \geq \sigma .
$$

Thus, for any function $f: E \longrightarrow \mathbb{R}$, we have

$$
\|\mathcal{E} f\|_{C^{m}\left(\mathbb{R}^{n}\right)} \leq A\|f\|_{C^{m}\left(E, \sigma_{\epsilon}\right)}, \quad \text { and }
$$

(14.9) $|\mathcal{E} f(x)-f(x)| \leq A\|f\|_{C^{m}\left(E, \sigma_{\epsilon}\right)} \cdot \sigma_{\epsilon}(x)$ for all $x \in E \cap B\left(y^{0}, c^{\prime}\right)$.

Here $A$ and $c^{\prime}$ depend only on $m$ and $n$. From (14.5), (14.6), (14.9), we have

$$
|\mathcal{E} f(x)-f(x)| \leq A \epsilon \cdot\|f\|_{C^{m}\left(E, \sigma_{\epsilon}\right)}
$$

for all $f$ and for all $x \in E_{0} \cap B\left(y^{0}, c^{\prime}\right)$.

We define a linear operator $L: C^{m}\left(E, \sigma_{\epsilon}\right) \longrightarrow C^{0}(E)$ by setting

$$
L f(x)=\left\{\begin{array}{ll}
f(x)-\mathcal{E} f(x) & \text { for } x \in E_{0} \cap B\left(y^{0}, c^{\prime}\right) \\
0 & \text { for all other } x \in E
\end{array} .\right.
$$

We then define the linear operator $\tilde{\mathcal{E}}: C^{m}\left(E, \sigma_{\epsilon}\right) \longrightarrow C^{m}\left(\mathbb{R}^{n}\right)$, by setting

$$
\tilde{\mathcal{E}} f=\mathcal{E} f+\mathcal{E}^{\text {trivial }}(L f) \text { for all } f \text {. }
$$

Note that

$$
\begin{aligned}
& \|\tilde{\mathcal{E}} f\|_{C^{m}\left(\mathbb{R}^{n}\right)} \leq\|\mathcal{E} f\|_{C^{m}\left(\mathbb{R}^{n}\right)}+\Gamma(E) \cdot\|L f\|_{C^{0}(E)} \quad(\text { see }(14.3)) \\
& \leq A\|f\|_{C^{m}\left(E, \sigma_{\epsilon}\right)}+\Gamma(E) \cdot\|L f\|_{C^{0}(E)} \quad(\text { see }(14.8)) \\
& \leq A\|f\|_{C^{m}\left(E, \sigma_{\epsilon}\right)}+\Gamma(E) \cdot\left[A \epsilon \cdot\|f\|_{C^{m}\left(E, \sigma_{\epsilon}\right)}\right] \quad(\text { see }(14.10),(14.11)) \\
& \leq 2 A \cdot\|f\|_{C^{m}\left(E, \sigma_{\epsilon}\right)}
\end{aligned}
$$

provided we take

$$
\epsilon<\frac{1}{\Gamma(E)}
$$


Let $x \in E_{1} \cap B\left(y^{0}, c^{\prime}\right)$. Then we have $L f(x)=0$ by definition (14.11), hence $\mathcal{E}^{\text {trivial }}(L f)(x)=0$ by (14.2). Consequently, (14.9) and (14.12) show that

$|\tilde{\mathcal{E}} f(x)-f(x)|=|\mathcal{E} f(x)-f(x)| \leq A\|f\|_{C^{m}\left(E, \sigma_{\epsilon}\right)} \cdot \sigma_{\epsilon}(x)=A\|f\|_{C^{m}\left(E, \sigma_{\epsilon}\right)} \cdot \sigma(x)$.

(See (14.6).) Thus,

$$
|\tilde{\mathcal{E}} f(x)-f(x)| \leq A\|f\|_{C^{m}\left(E, \sigma_{\epsilon}\right)} \cdot \sigma(x)
$$

for all $f$, and for all $x \in E_{1} \cap B\left(y^{0}, c^{\prime}\right)$.

On the other hand, suppose $x \in E_{0} \cap B\left(y^{0}, c^{\prime}\right)$. Then (14.11) gives $L f(x)=f(x)-\mathcal{E} f(x)$, hence $(14.2)$ yields $\mathcal{E}^{\text {trivial }}(L f)(x)=f(x)-\mathcal{E} f(x)$; and therefore (14.12) implies $\tilde{\mathcal{E}} f(x)=f(x)$. Thus, we have

$$
|\tilde{\mathcal{E}} f(x)-f(x)| \leq A\|f\|_{C^{m}\left(E, \sigma_{\epsilon}\right)} \cdot \sigma(x) \quad \text { (both sides vanish) }
$$

for all $f$, and for all $x \in E_{0} \cap B\left(y^{0}, c^{\prime}\right)$.

From (14.4), (14.15), (14.16), we conclude that

$$
|\tilde{\mathcal{E}} f(x)-f(x)| \leq A\|f\|_{C^{m}\left(E, \sigma_{\epsilon}\right)} \cdot \sigma(x)
$$

for all $f$, and for all $x \in E \cap B\left(y^{0}, c^{\prime}\right)$.

From (14.7), (14.13), (14.17), we obtain the following:

$$
\begin{aligned}
\|\tilde{\mathcal{E}} f\|_{C^{m}\left(\mathbb{R}^{n}\right)} & \leq 2 A\|f\|_{C^{m}(E, \sigma)} \quad \text { for all } f ; \text { and } \\
|\tilde{\mathcal{E}} f(x)-f(x)| & \leq A\|f\|_{C^{m}(E, \sigma)} \cdot \sigma(x)
\end{aligned}
$$

for all $f$ and for all $x \in E \cap B\left(y^{0}, c^{\prime}\right)$.

Since $A$ and $c^{\prime}$ depend only on $m$ and $n$, the conclusions of the Local Theorem 1 are immediate from (14.18) and (14.19). The proof of the Lemma is complete.

It is now easy to finish the proof of Theorem 1 . Let $E \subset \mathbb{R}^{n}$ be finite, and let $\sigma: E \longrightarrow[0, \infty)$ be given.

For each $y \in \mathbb{R}^{n}$, we obtain from Lemma 1 above a linear operator $\mathcal{E}^{y}: C^{m}(E, \sigma) \longrightarrow C^{m}\left(\mathbb{R}^{n}\right)$, satisfying for each $f \in C^{m}(E, \sigma)$ the estimates

$$
\left\|\mathcal{E}^{y} f\right\|_{C^{m}\left(\mathbb{R}^{n}\right)} \leq A\|f\|_{C^{m}(E, \sigma)} \quad \text { and }
$$

(14.21) $\left|\mathcal{E}^{y} f(x)-f(x)\right| \leq A\|f\|_{C^{m}(E, \sigma)} \cdot \sigma(x)$ for all $x \in B\left(y, c^{\prime}\right) \cap E$.

Here $A, c^{\prime}$ depend only on $m$ and $n$. For the rest of this section, we use $c, C, C^{\prime}$, etc., to denote constants depending only on $m$ and $n$. 
We introduce a partition of unity

$$
\sum_{\nu} \theta_{\nu}(x)=1 \quad \text { for all } x \in \mathbb{R}^{n}
$$

where

(14.22a) $\left\|\theta_{\nu}\right\|_{C^{m}\left(\mathbb{R}^{n}\right)} \leq C$,

(14.23) $0 \leq \theta_{\nu} \leq 1$ on $\mathbb{R}^{n}$

(14.24) $\operatorname{supp} \theta_{\nu} \subset B\left(y_{\nu}, \frac{1}{2} c^{\prime}\right) \quad$ with $c^{\prime}$ as in (14.21), and

(14.25) no point of $\mathbb{R}^{n}$ belongs to more than $C$ of the balls $B\left(y_{\nu}, c^{\prime}\right)$.

For $f \in C^{m}(E, \sigma)$, we define

$$
\mathcal{E} f=\sum_{\nu} \theta_{\nu} \cdot\left(\mathcal{E}^{y_{\nu}} f\right)
$$

We have

$$
\begin{aligned}
&\|\mathcal{E} f\|_{C^{m}\left(\mathbb{R}^{n}\right)} \leq C \cdot \sup _{\nu}\left\|\theta_{\nu} \cdot\left(\mathcal{E}^{y_{\nu}} f\right)\right\|_{C^{m}\left(\mathbb{R}^{n}\right)} \\
& \quad(\operatorname{by}(14.24),(14.25),(14.26)) \\
& \leq C^{\prime} \sup _{\nu}\left\|\mathcal{E}^{y_{\nu}} f\right\|_{C^{m}\left(\mathbb{R}^{n}\right)} \quad(\text { see }(14.22 \mathrm{a})) \\
& \leq C^{\prime \prime}\|f\|_{C^{m}(E, \sigma)} \quad(\text { see }(14.20)) .
\end{aligned}
$$

Also, for $x \in E \cap B\left(y_{\nu}, c^{\prime}\right)$, we have

$$
\left|\theta_{\nu}(x) \mathcal{E}^{y_{\nu}} f(x)-\theta_{\nu}(x) f(x)\right| \leq A\|f\|_{C^{m}(E, \sigma)} \cdot \theta_{\nu}(x) \sigma(x)
$$

thanks to (14.21) and (14.23).

On the other hand, for $x \in E \backslash B\left(y_{\nu}, c^{\prime}\right)$, (14.28) still holds, since both sides are zero, thanks to (14.24). Thus (14.28) holds for all $x \in E$. Summing (14.28) over $\nu$, and recalling (14.22) and (14.26), we find that

$$
|\mathcal{E} f(x)-f(x)| \leq A\|f\|_{C^{m}(E, \sigma)} \cdot \sigma(x)
$$

for all $f \in C^{m}(E, \sigma)$ and all $x \in E$.

Since $C^{\prime \prime}$ and $A$ depend only on $m$ and $n$, estimates (14.27) and (14.29) are the conclusions of Theorem 1 . The proof of Theorem 1 is complete. 


\section{Banach Limits}

In this section, we recall the basic properties of Banach limits.

A directed set is a set $\mathcal{D}$ with a partial order $>$, with the property that, given any $E_{1}, E_{2} \in \mathcal{D}$, there exists $E \in \mathcal{D}$, with $E \geq E_{1}$ and $E \geq E_{2}$.

Let $\mathcal{D}$ be a directed set. A $\mathcal{D}$-sequence is a function from $\mathcal{D}$ to the real numbers. We denote $\mathcal{D}$-sequences by $\vec{\xi}=\left(\xi_{E}\right)_{E \in \mathcal{D}}$.

We write $C^{\circ}(\mathcal{D})$ to denote the vector space of bounded $\mathcal{D}$-sequences, equipped with the sup norm.

From a well-known application of the Hahn-Banach theorem (see, eg. [7]), there exists a linear functional $\ell_{\mathcal{D}}: C^{\circ}(\mathcal{D}) \longrightarrow \mathbb{R}$, satisfying the estimate

$$
\liminf _{E \longrightarrow \infty} \xi_{E} \leq \ell_{\mathcal{D}}(\vec{\xi}) \leq \limsup _{E \longrightarrow \infty} \xi_{E} \quad \text { for all } \vec{\xi}=\left(\xi_{E}\right)_{E \in \mathcal{D}} \in C^{\circ}(\mathcal{D}) .
$$

Here,

$$
\liminf _{E \longrightarrow \infty} \xi_{E}=\sup _{\tilde{E} \in \mathcal{D}}\left(\inf _{E \geq \tilde{E}} \xi_{E}\right), \quad \text { and } \quad \limsup _{E \longrightarrow \infty} \xi_{E}=\inf _{\tilde{E} \in \mathcal{D}}\left(\sup _{E \geq \tilde{E}} \xi_{E}\right) .
$$

The functional $\ell_{\mathcal{D}}$ is far from unique, but we fix some $\ell_{\mathcal{D}}$ as above, and call it a Banach limit.

\section{Equivalence of Norms for Finite Sets}

In this section, we prove the following straightforward result.

Lemma ENFS. Let $E \subset \mathbb{R}^{n}$ be finite, and let $\sigma: E \longrightarrow[0, \infty)$. Then, for each $f: E \longrightarrow \mathbb{R}$, we have

$$
c\|f\|_{C^{m}(E, \sigma)} \leq\|f\|_{C^{m-1,1}(E, \sigma)} \leq C\|f\|_{C^{m}(E, \sigma)},
$$

with $c$ and $C$ depending only on $m$ and $n$.

Proof. The second estimate is immediate from the definitions and the fact that $\|F\|_{C^{m-1,1}\left(\mathbb{R}^{n}\right)} \leq C\|F\|_{C^{m}\left(\mathbb{R}^{n}\right)}$ for any $F \in C^{m}\left(\mathbb{R}^{n}\right)$. Here and throughout this proof, $c, C$, etc. stand for constants determined by $m$ and $n$.

To prove the first estimate in (16.1), we may assume that

$$
\|f\|_{C^{m-1,1}(E, \sigma)}=1 \text {. }
$$

We must then show that

$$
\|f\|_{C^{m}(E, \sigma)} \leq C
$$


In view of $(16.2)$, there exists $F \in C^{m-1,1}\left(\mathbb{R}^{n}\right)$, with

$$
\begin{aligned}
\|F\|_{C^{m-1,1}\left(\mathbb{R}^{n}\right)} & \leq C, \quad \text { and } \\
|F(x)-f(x)| & \leq C \sigma(x) \text { for all } x \in E .
\end{aligned}
$$

By convolving $F$ with an approximate identity, we obtain a family of functions $F_{\delta} \in C^{m}\left(\mathbb{R}^{n}\right)$, parametrized by $\delta>0$, with the following properties:

$$
\begin{aligned}
& \left\|F_{\delta}\right\|_{C^{m}\left(\mathbb{R}^{n}\right)} \leq C\|F\|_{C^{m-1,1}\left(\mathbb{R}^{n}\right)} \leq C^{\prime} \quad(\text { see }(16.4)) ; \\
& F_{\delta} \longrightarrow F \text { pointwise, as } \delta \longrightarrow 0 .
\end{aligned}
$$

Let $\epsilon>0$ be small enough, to be picked later. Since (16.7) holds and $E$ is finite, we may pick $\delta>0$ small enough so that we have

$$
\left|F_{\delta}(x)-F(x)\right| \leq \epsilon \quad \text { for all } x \in E .
$$

From now on, we fix $\delta$ satisfying (16.8) (and depending on $\epsilon$, of course). From (16.5) and (16.8), we get

$$
\left|F_{\delta}(x)-f(x)\right| \leq C \sigma(x)+\epsilon \text { for all } x \in E .
$$

On the other hand, since $E$ is finite, we have the following trivial remark:

(16.10) Given a function $g \in C^{0}(E)$, there exists $G \in C^{m}\left(\mathbb{R}^{n}\right)$, with

(a) $G(x)=g(x)$ for all $x \in E$, and

(b) $\|G\|_{C^{m}\left(\mathbb{R}^{n}\right)} \leq \Gamma(E) \cdot\|g\|_{C^{0}(E)}$,

for a finite constant $\Gamma(E)$ depending on $E$. In view of (16.9), there exists a function $g: E \longrightarrow \mathbb{R}$, with

$$
\begin{aligned}
& |g(x)| \leq \epsilon \quad \text { for all } x \in E, \text { and } \\
& \left|\left(F_{\delta}(x)-f(x)\right)-g(x)\right| \leq C \sigma(x) \quad \text { for all } x \in E .
\end{aligned}
$$

Applying (16.10) to the function $g$ in (16.11), (16.12), we obtain a function $G \in C^{m}\left(\mathbb{R}^{n}\right)$, with the following properties:

$$
\begin{aligned}
& \|G\|_{C^{m}\left(\mathbb{R}^{n}\right)} \leq \Gamma(E) \cdot \epsilon ; \quad \text { and } \\
& \left|F_{\delta}(x)-f(x)-G(x)\right| \leq C \sigma(x) \text { for all } x \in E .
\end{aligned}
$$

We pick $\epsilon<1 / \Gamma(E)$, and set $\tilde{F}=F_{\delta}-G$. From (16.6) and (16.13), we see that

$$
\|\tilde{F}\|_{C^{m}\left(\mathbb{R}^{n}\right)} \leq C^{\prime}
$$

From (16.14) we have

$$
|\tilde{F}(x)-f(x)| \leq C \sigma(x) \quad \text { for all } x \in E .
$$

Estimates (16.15) and (16.16) prove (16.3), thus completing the proof of the Lemma. 


\section{Proof of Theorem 2}

We assume here that $m \geq 2$, leaving to the reader the task of modifying our arguments for the case $m=1$.

Let $E \subset \mathbb{R}^{n}$, and let $\sigma: E \longrightarrow[0, \infty)$ be given. Let $\mathcal{D}$ denote the set of all finite subsets $E_{1} \subset E$, partially ordered by inclusion: $E_{1} \leq E_{2}$ if and only if $E_{1} \subseteq E_{2}$. Thus $\mathcal{D}$ is a directed set. For each $E_{1} \in \mathcal{D}$, we apply Theorem 1, together with Lemma ENFS in Section 16, to obtain a linear operator $\mathcal{E}\left[E_{1}\right]: C^{m-1,1}\left(E_{1},\left.\sigma\right|_{E_{1}}\right) \longrightarrow C^{m}\left(\mathbb{R}^{n}\right)$, with

$$
\left\|\mathcal{E}\left[E_{1}\right] f\right\|_{C^{m}\left(\mathbb{R}^{n}\right)} \leq C\|f\|_{C^{m-1,1}\left(E_{1},\left.\sigma\right|_{E_{1}}\right)}
$$

and

$$
\left|\left(\mathcal{E}\left[E_{1}\right] f\right)(x)-f(x)\right| \leq C \sigma(x) \cdot\|f\|_{C^{m-1,1}\left(E_{1},\left.\sigma\right|_{E_{1}}\right)} \text { on } E_{1} \text {, for all } f \text {. }
$$

Here, and throughout this section, $c, C, C^{\prime}$, etc., denote constants depending only on $m$ and $n$.

Note that (17.1) shows in particular that

$$
\sup _{E_{1} \in \mathcal{D}}\left|\partial^{\beta}\left(\mathcal{E}\left[E_{1}\right] f\right)(x)\right| \leq C\|f\|_{C^{m-1,1}(E, \sigma)}
$$

for all $f \in C^{m-1,1}(E, \sigma),|\beta| \leq m, x \in \mathbb{R}^{n}$.

We define an element $\vec{\xi}(f, \beta, x) \in C^{\circ}(\mathcal{D})$, by setting

$$
\vec{\xi}(f, \beta, x)=\left(\partial^{\beta}\left(\mathcal{E}\left[E_{1}\right] f\right)(x)\right)_{E_{1} \in \mathcal{D}} .
$$

In view of (17.3), we have $\vec{\xi}(f, \beta, x) \in C^{\circ}(\mathcal{D})$, and

$$
\|\vec{\xi}(f, \beta, x)\|_{C^{\circ}(\mathcal{D})} \leq C\|f\|_{C^{m-1,1}(E, \sigma)},
$$

for $f \in C^{m-1,1}(E, \sigma),|\beta| \leq m, x \in \mathbb{R}^{n}$.

Applying the Banach limit $\ell_{\mathcal{D}}$ to $\vec{\xi}(f, \beta, x)$, we obtain functions $F_{\beta}(x)$, defined by

$$
F_{\beta}(x)=\ell_{\mathcal{D}}(\vec{\xi}(f, \beta, x)) \text { for }|\beta| \leq m, x \in \mathbb{R}^{n},
$$

with $f \in C^{m-1,1}(E, \sigma)$ given. Note that the map

$$
\mathcal{E}: f \longrightarrow F_{0} \text { is linear. }
$$

(Here, 0 denotes the zero multi-index.) We will show that $\mathcal{E}$ satisfies the conclusions of Theorem 2 . 
First, we establish the smoothness of $F_{0}$. Immediately from (17.5), (17.6) and the properties of the Banach limit, we have

$$
\sup _{x \in \mathbb{R}^{n}}\left|F_{\beta}(x)\right| \leq C\|f\|_{C^{m-1,1}(E, \sigma)} \text { for }|\beta| \leq m .
$$

Moreover, for $x, y \in \mathbb{R}^{n},|\beta| \leq m-1$, and $E_{1} \in \mathcal{D}$, estimate (17.1) gives

$$
\left|\partial^{\beta}\left(\mathcal{E}\left[E_{1}\right] f\right)(x)-\partial^{\beta}\left(\mathcal{E}\left[E_{1}\right] f\right)(y)\right| \leq C|x-y| \cdot\|f\|_{C^{m-1,1}(E, \sigma)} .
$$

Together with (17.4), this shows that

$$
\|\vec{\xi}(f, \beta, x)-\vec{\xi}(f, \beta, y)\|_{C^{\circ}(\mathcal{D})} \leq C|x-y| \cdot\|f\|_{C^{m-1,1}(E, \sigma)} .
$$

Taking the Banach limit, and recalling (17.6), we see that

$$
\left|F_{\beta}(x)-F_{\beta}(y)\right| \leq C|x-y| \cdot\|f\|_{C^{m-1,1}(E, \sigma)} \quad \text { for }|\beta| \leq m-1, x, y \in \mathbb{R}^{n} \text {. }
$$

Similarly, suppose $x, y \in \mathbb{R}^{n}\left(y=\left(y_{1}, \ldots, y_{n}\right)\right)$, and let $|\beta| \leq m-2$. For $j=1, \ldots, n$, let $\beta[j]$ denote the sum of $\beta$ and the $j^{\text {th }}$ unit multi-index. Then (17.1) and Taylor's theorem show that

$$
\begin{array}{r}
\left|\partial^{\beta}\left(\mathcal{E}\left[E_{1}\right] f\right)(x+y)-\partial^{\beta}\left(\mathcal{E}\left[E_{1}\right] f\right)(x)-\sum_{j=1}^{n}\left[\partial^{\beta[j]}\left(\mathcal{E}\left[E_{1}\right] f\right)(x)\right] y_{j}\right| \leq \\
\leq C|y|^{2}\|f\|_{C^{m-1,1}(E, \sigma)}
\end{array}
$$

for all $E_{1} \in \mathcal{D}$. That is,

$$
\begin{aligned}
\| \vec{\xi}(f, \beta, x+y)-\vec{\xi}(f, \beta, x)- & \sum_{j=1}^{n} y_{j} \vec{\xi}(f, \beta[j], x) \|_{C^{\circ}(\mathcal{D})} \leq \\
\leq C|y|^{2}\|f\|_{C^{m-1,1}(E, \sigma)} & \quad \text { (see }(17.4)) .
\end{aligned}
$$

Applying the Banach limit and recalling (17.6), we find that

$$
\left|F_{\beta}(x+y)-F_{\beta}(x)-\sum_{j=1}^{n} y_{j} F_{\beta[j]}(x)\right| \leq C|y|^{2}\|f\|_{C^{m-1,1}(E, \sigma)}
$$

for $x \in \mathbb{R}^{n}, y=\left(y_{1}, \ldots, y_{n}\right) \in \mathbb{R}^{n}, f \in C^{m-1,1}(E, \sigma),|\beta| \leq m-2$.

This shows that

$$
F_{\beta} \text { is differentiable, and } \frac{\partial}{\partial x_{j}} F_{\beta}=F_{\beta[j]},
$$

for $|\beta| \leq m-2$ and $j=1, \ldots, n$. From (17.8), (17.9), (17.10), we conclude that $F_{0} \in C^{m-1,1}\left(\mathbb{R}^{n}\right)$, and

$$
\left\|F_{0}\right\|_{C^{m-1,1}\left(\mathbb{R}^{n}\right)} \leq C\|f\|_{C^{m-1,1}(E, \sigma)} .
$$

Thus, we have established the smoothness of $F_{0}$. Next, we estimate $\mid F_{0}(x)-$ $f(x) \mid$ for $x \in E$. 
For $\tilde{x} \in E$, and let $\tilde{E}_{1}=\{\tilde{x}\} \in \mathcal{D}$. If $E_{1} \in \mathcal{D}$ and $E_{1} \geq \tilde{E}_{1}$, then $\tilde{x} \in E_{1}$, hence (17.2) implies that

$$
\left|\left(\mathcal{E}\left[E_{1}\right] f\right)(\tilde{x})-f(\tilde{x})\right| \leq C \sigma(\tilde{x}) \cdot\|f\|_{C^{m-1,1}(E, \sigma)} .
$$

Consequently, we have

$$
\begin{aligned}
& \limsup _{E_{1} \longrightarrow \infty}\left(\mathcal{E}\left[E_{1}\right] f\right)(\tilde{x}) \leq f(\tilde{x})+C \sigma(\tilde{x}) \cdot\|f\|_{C^{m-1,1}(E, \sigma)}, \quad \text { and } \\
& \liminf _{E_{1} \longrightarrow \infty}\left(\mathcal{E}\left[E_{1}\right] f\right)(x) \geq f(\tilde{x})-C \sigma(\tilde{x}) \cdot\|f\|_{C^{m-1,1}(E, \sigma)} .
\end{aligned}
$$

Also, from (17.4), (17.6) with $\beta=0$, and from the properties of the Banach limit, we have

$$
\liminf _{E_{1} \longrightarrow \infty}\left(\mathcal{E}\left[E_{1}\right] f\right)(\tilde{x}) \leq F_{0}(\tilde{x}) \leq \limsup _{E_{1} \longrightarrow \infty}\left(\mathcal{E}\left[E_{1}\right] f\right)(\tilde{x}) .
$$

Inequalities (17.12), (17.13), (17.14) show that

$$
\left|F_{0}(\tilde{x})-f(\tilde{x})\right| \leq C \sigma(\tilde{x}) \cdot\|f\|_{C^{m-1,1}(E, \sigma)} .
$$

We have proven (17.15) for all $\tilde{x} \in E$ and $f \in C^{m-1,1}(E, \sigma)$.

Our estimates (17.11), (17.15) show that the linear operator $\mathcal{E}$ in (17.7) maps $C^{m-1,1}(E, \sigma)$ to $C^{m-1,1}\left(\mathbb{R}^{n}\right)$, and satisfies the conclusions of Theorem 2. The proof of Theorem 2 is complete.

\section{References}

[1] Bierstone, E., Milman, P. and Pawlucki, W.: Differentiable functions defined on closed sets. A problem of Whitney. Invent. Math. 151 (2003), no. 2, 329-352.

[2] Brudnyi, Y.: A certain extension theorem. Funkcional Anal. i Prilzhen. 4 (1970), no. 3, 97-98. English translation in Func. Anal. Appl. 4 (1970), $252-253$.

[3] Brudnyi Y., and Shvartsman, P.: A linear extension operator for a space of smooth functions defined on closed subsets of $\mathbb{R}^{n}$. Dokl. Akad. Nauk SSSR 280 (1985), no. 2, 268-272.

[4] Brudnyi, Y. and Shvartsman, P.: Generalizations of Whitney's extension theorem. International Math. Res. Notices 3 (1994), 129-139.

[5] Brudnyi Y., and Shvartsman, P.: The Whitney problem of existence of a linear extension operator. J. Geom. Anal. 7 (1997), no. 4, 515-574.

[6] Brudnyi Y., And Shvartsman, P.: Whitney's extension problem for multivariate $C^{1, \omega}$ functions. Trans. Amer. Math. Soc. 353 (2001), no. 6, $2487-2512$. 
[7] Dunford, N. and Schwartz, J. T.: Linear Operators vol I. Pure and Applied Mathematics 7. Interscience Publishers, J. Wiley \& Sons, New York, 1964.

[8] Fefferman, C.: A sharp form of Whitney's extension theorem. Ann. of Math. (2) 161 (2005), no. 1, 507-575.

[9] Glaeser, G.: Études de quelques algèbres tayloriennes. J. Analyse Math. 6 (1958), 1-124.

[10] Guzmán, M. DE: Differentiation of Integrals in $\mathbb{R}^{n}$. Lecture Notes in Mathematics 481. Springer Verlag, Berlin-New York, 1975.

[11] Malgrange, B.: Ideals of Differentiable Functions. Oxford University Press, Oxford, 1966.

[12] Shvartsman, P.: Lipschitz selections of multivalued mappings and traces of the Zygmund class of functions to an arbitrary compact. Dokl. Acad. Nauk SSSR 276 (1984), no. 3, 559-562. English translation in Soviet Math. Dokl. 29 (1984), 565-568.

[13] Shvartsman, P.: Traces of functions of Zygmund classes. Sibirsk. Mat. Zh. 28 (1987), no. 5, 203-215. English translation in Siberian Math. J. 28 (1987), 853-863.

[14] Shvartsman, P.: Lipschitz selections of set-valued functions and Helly's theorem. J. Geom. Anal. 12 (2002), no. 2, 289-324.

[15] Stein, E. M.: Singular Integrals and Differentiability Properties of Functions. Princeton Univ. Press, Princeton, 1970.

[16] Webster, R.: Convexity. Oxford Science Publications. The Clarendon Press, Oxford University Press, New York, 1994.

[17] Whitney, H.: Analytic extensions of differentiable functions defined in closed sets. Trans. Amer. Math. Soc. 36 (1934), no. 1, 63-89.

[18] Whitney, H.: Differentiable functions defined in closed sets I. Trans. Amer. Math. Soc. 36 (1934), no. 2, 369-387.

[19] Whitney, H.: Functions differentiable on the boundaries of regions. Ann. of Math. (2) 35 (1934), no. 3, 482-485.

Recibido: 21 de agosto de 2003.

Charles Fefferman

Department of Mathematics

Princeton University

Fine Hall, Washington Road

Princeton, New Jersey 08544, USA cf@math.princeton.edu

Partially supported by NSF Grant DMS 0070692. It is a pleasure to thank Gerree Pecht for expertly TeXing this paper. 